\title{
An Overview on Nutritional Aspects of Plant-Based Beverages Used as Substitutes for Cow's Milk
}

\author{
Isabel Fructuoso ${ }^{1}$, Bernardo Romão ${ }^{1}$, Heesup Han ${ }^{2} * \mathbb{D}$, António Raposo ${ }^{3} * \mathbb{D}$, Antonio Ariza-Montes ${ }^{4} \mathbb{D}$, \\ Luis Araya-Castillo ${ }^{5}$ and Renata Puppin Zandonadi ${ }^{1}$ (D)
}

1 Department of Nutrition, University of Brasília, Brasília 70910-900, Brazil; belfructuoso@gmail.com (I.F.); bernardolima156@gmail.com (B.R.); renatapz@unb.br (R.P.Z.)

2 College of Hospitality and Tourism Management, Sejong University, 98 Gunja-dong, Gwanjin-gu, Seoul 143-747, Korea

3 CBIOS (Research Center for Biosciences and Health Technologies), Universidade Lusófona de Humanidades e Tecnologias, Campo Grande 376, 1749-024 Lisboa, Portugal

4 Social Matters Research Group, Universidad Loyola Andalucía, C/Escritor Castilla Aguayo, 414004 Córdoba, Spain; ariza@uloyola.es

5 Facultad de Economía y Negocios, Universidad Andrés Bello, Santiago de Chile 7591538, Chile; luis.araya@unab.cl

* Correspondence: heesup.han@gmail.com (H.H.); antonio.raposo@ulusofona.pt (A.R.)

Citation: Fructuoso, I.; Romão, B.; Han, H.; Raposo, A.; Ariza-Montes, A.; Araya-Castillo, L.; Zandonadi, R.P. An Overview on Nutritional Aspects of Plant-Based Beverages Used as Substitutes for Cow's Milk. Nutrients 2021, 13, 2650. https://doi.org/ $10.3390 /$ nu13082650

Academic Editor: Alaa El-Din A. Bekhit

Received: 1 July 2021

Accepted: 27 July 2021

Published: 30 July 2021

Publisher's Note: MDPI stays neutra with regard to jurisdictional claims in published maps and institutional affiliations.

Copyright: (c) 2021 by the authors Licensee MDPI, Basel, Switzerland. This article is an open access article distributed under the terms and conditions of the Creative Commons Attribution (CC BY) license (https:// creativecommons.org/licenses/by/ $4.0 /)$.

\begin{abstract}
The presence of milk in meals and products consumed daily is common and at the same time the adoption of a milk-free diet increases due to milk allergy, lactose intolerance, vegan diets, and others. Therefore, there is an increasing demand for plant-based beverages, which present variable and, sometimes, unknown nutritional characteristics. This study sought to compare the nutritional aspects of plant-based beverages used as substitutes for cow's milk described in scientific studies. Therefore, we used a review of the scientific literature on PubMed, Google Scholar, Scopus, Web of Science, Google Patents, Embase, and ScienceDirect databases. The inclusion criteria were scientific studies referring to plant-based beverage used as an alternative to cow's milk; published in the English language; present data on the serving size, ingredients, and nutritional composition, containing at least data on energy and macronutrients of plant-based beverages. Ingredients and data on energy, macronutrients, and, if available, dietary fiber and some micronutrients of plant-based beverages were collected. Data were obtained from 122 beverages of 22 different matrices, with soy being the most used $(27.87 \%, n=34)$. The variation in the amount of nutrients found was 6-183 Kcal/100 mL for energy value; 0.00-22.29 g/100 mL for carbohydrate; 0.06-12.43 g/100 mL for protein; 0.00-19.00 g/100 mL for lipid; 0.00-4.40 g/100 mL for dietary fiber; 0.00-1252.94 mg/100 mL for calcium; 0.04-1.40 mg/100 mL for iron; 0.84-10,178.60 mg/100 mL for magnesium; $0.00-343.43 \mathrm{mg} / 100 \mathrm{~mL}$ for sodium. Salt was the most commonly found added ingredient in plant-based beverages. Some beverages have reached certain amounts of cow's milk nutrients. However, studies have pointed out differences in their qualities/types. Thus, attention is needed when replacing milk with these alternatives.
\end{abstract}

Keywords: plant-based beverage; nutritional compositional; added ingredients; energy; macronutrients; dietary fiber; micronutrients

\section{Introduction}

Milk is the "lacteal secretion, practically free from colostrum, obtained by the complete milking of one or more healthy cows" [1]. Worldwide, cow's milk and its derivatives are consumed by more than 6 billion people [2], on average of $116.50 \mathrm{~kg} /$ inhabitant/year [3], standing out mainly due to its content of high biological value proteins and calcium [4-6].

Despite the dietary benefits provided by milk, some people present milk-related disorders as cow's milk allergy (CMA), which reaches about $0.50 \%$ and $3.50 \%$ of individuals [6-8], and lactose intolerance (65.00-75.00\% of individuals) [6,9-12]. Additionally, some 
people choose to follow a milk-free diet like some types of vegetarians and vegans [13]. Given the high presence of milk in meals and products consumed daily and people who follow a milk-free diet, it is necessary to seek alternatives to replace this product in daily meals [14].

Regarding CMA, some animal-based alternatives may be used to replace cow's milk, such as donkey and goat milk [15-17]. Nevertheless, an altered version of cow's milk, the A2A2 kind where cow's milk main protein A1 $\beta$-casein is modified to a non-allergenic version, the A2 $\beta$-casein type, can also be used as an alternative with good results [18]. Though, despite the replacement of the main animal protein, these options often present lactose, which may be an impairment to a considerable part of the population [12].

Considering all milk-related disorders, the main alternatives used to replace milk are water-soluble extracts of legumes (e.g., soybean (Glycine max) and chickpea (Cicer arietinum)), nuts (e.g., almond (Prunus dulcis), cashew nut (Anacardium occidentale), hazelnut Corylus avellana), and Brazil nut (Bertholletia excelsa), seeds (e.g., sunflower (Helianthus annus) and sesame (Sesamum indicum)), cereals (e.g., rice (Oryza spp.) and oat (Avena sativa)) or pseudocereals (e.g., quinoa (Chenopodium quinoa)) [5,19-21]. It is important to highlight that consumers search for a product to be used as cow's milk substitute with similar sensorial aspects regarding color, texture and, when possible, flavor [22]. However, frequently, milk substitutes' nutritional characteristics are not similar to cow's milk [10].

Plant-based beverages present the composition variable on the amount of macro and micronutrients and the presence of bioactive compounds and antinutritional factors [10]. As for bioactive compounds, for example, the soy-based beverage contains isoflavones and phytosterols; almond-based beverage contains $\alpha$-tocopherol and arabinose; oat-based beverage contains $\beta$-glucan. Regarding antinutritional factors, for example, there is the presence of oxalates and phytates in the sesame-based beverage and phytates in the oatbased beverage. Technological interventions are being studied to improve these beverages' sensory acceptability and nutritional quality $[10,19]$. Despite that, we hypothesized that plant-based beverages are lower in protein and calcium than cow's milk, but some of them are better than others regarding nutritional quality.

The demand for plant-based beverages has been increasing worldwide over the years $[6,10,11,14,23-25]$ due to their potential health benefits $[14,19]$ and the growing adoption of a milk-free diet. It is estimated an annual growth rate of $10.18 \%$ between 2020 and 2024 [26].

Considering the scarcity of studies comparing the nutritional composition of different milk substitutes to guide the population about the best alternative to compose their diet, this study sought to compare the nutritional aspects of plant-based beverages used as substitutes for cow's milk described in scientific studies.

\section{Materials and Methods}

\subsection{Data Collection}

Data were collected on plant-based beverages from publications in scientific journals without the geographical limitations of the studies. PubMed, Google Scholar, Scopus, Web of Science, Google Patents, Embase, and ScienceDirect databases were searched and the search terms used are available in Appendix A.

\subsubsection{Inclusion and Exclusion Criteria}

The inclusion criteria were scientific studies referring to plant-based beverage used as an alternative to cow's milk; have been published in English; present data on the serving size, ingredients, and nutritional composition, containing at least data on energy (kcal) and macronutrients (carbohydrates, protein, and lipids) of plant-based beverages.

\subsubsection{Collected Information}

The data collected from scientific studies were authors; country of origin; year of publication; types of plant-based beverages presented; serving size of the beverages analyzed; 
nutritional composition; ingredients; specifications on the origin of the beverages, that is, if the beverage was prepared and analyzed for the study or if it is a beverage available on the market and had its nutritional values removed from the label, among other information. Regarding nutritional composition, the values collected from plant-based beverages were energy value; macronutrients (carbohydrates, protein, and lipids); if available, dietary fiber, calcium, iron, magnesium, and sodium.

The nutrient values were converted to the equivalent of $100 \mathrm{~mL}$ using Microsoft Excel ${ }^{\circledR}$. For the serving sizes in grams, a density of $1.027 \mathrm{~g} / \mathrm{mL}$ was considered, based on the soy-based beverage values, and the calculations were made [27]. When available, salt values were converted into the equivalent in sodium, given that $1 \mathrm{~g}$ of salt presents $400 \mathrm{mg}$ of sodium [28]. Microsoft Excel ${ }^{\circledR}$ was used to standardize data.

\subsection{Data Processing}

With the worldclouds platform's help, a word cloud was generated with the ingredients excluding water and the beverages' implemented matrix, for example, "almond" [29]. Names were represented according to their proportional frequencies among all ingredients in all analyzed samples, given that higher frequencies result in words with bigger sizes.

\section{Results}

A total of 32 studies were selected according to the inclusion and exclusion criteria. The studies were conducted in 13 different countries: Brazil $(59.38 \% ; n=19)$ [30-48]; India $(6.25 \% ; n=2)$ [49,50]; Argentina (3.13\%; $n=1)$ [51]; Belgium $(3.13 \% ; n=1)$ [52]; Canada $(3.13 \% ; n=1)$ [23]; Denmark $(3.13 \% ; n=1)$ [53]; Germany $(3.13 \% ; n=1)$ [54]; Ghana $(3.13 \%$; $n=1)$ [55]; Iran $(3.13 \% ; n=1)$ [56]; Ireland $(3.13 \% ; n=1)$ [57]; Nigeria $(3.13 \% ; n=1)$ [58]; Pakistan $(3.13 \% ; n=1)$ [59]; Turkey $(3.13 \% ; n=1)$ [60].

From these studies, data were obtained from 122 beverages made from 22 different matrices. Of these matrices, $18.18 \%(n=4)$ belong to the group of cereals that constituted a total of $18.85 \%(n=23)$ of beverages: rice (Oryza spp.) $(11.48 \%, n=14)[30,37,46,52-54,57,60]$; oat (Avena sativa) $(5.74 \%, n=7)[31,50,52-54,57]$; millet (Setaria italica) $(0.82 \%, n=1)$ [54]; spelt (Triticum spelta) $(0.82 \%, n=1)$ [54].

Concerning legumes, $13.64 \%(n=3)$ of the matrices are part of this group, totaling $29.51 \%(n=36)$ beverages: soy (Glycine max) $(27.87 \%, n=34)[23,38,42-44,48,52-56]$; baru almond (Dipteryx alata) $(0.82 \%, n=1)$ [40]; groundnut (Arachis hypogaea) $(0.82 \%, n=1)$ [49].

A total of $36.36 \%(n=8)$ of the matrices belong to the group of nuts that constituted $41.80 \%(n=51)$ of beverages: almond (Prunus dulcis) $(9.84 \%, n=12)[23,36,52-54,57,58]$; sapucaia nut (Lecythis pisonis) $(9.02 \%, n=11)$ [47]; cashew nut (Anacardium occidentale) $(6.56 \%$, $n=8)[23,32,33,52,54,57,59]$; coconut (Cocos nucifera) $(6.56 \%, n=8)$ [23,52-54,57]; hazelnut (Corylus avellana) $(3.28 \%, n=4)[53,54,57]$; cupuaçu almond (Theobroma grandiflorum) $(2.46 \%$, $n=3)$ [35]; tucumã almond (Astrocaryum vulgare) $(2.46 \%, n=3)$ [35]; macadamia nut (Macadamia spp.) $(1.64 \%, n=2)[54,57]$.

About pseudocereals, $9.09 \%(n=2)$ of the matrices are part of this group that were used to produce a total of $3.28 \%(n=4)$ of beverages: quinoa (Chenopodium quinoa) $(2.46 \%$, $n=3)$ [39,53,57]; amaranth (Amaranthus hypochondriacus) $(0.82 \%, n=1)$ [51]. Regarding seeds, $4(18.18 \%)$ of the matrices correspond to this group that generated a total of $5.74 \%$ $(n=7)$ of beverages: hemp (Cannabis sativa) $(2.46 \%, n=3)[23,53,57]$; sesame seed (Sesamum indicum) $(1.64 \%, n=2)$ [34]; safflower seed (Carthamus tinctorius) $(0.82 \%, n=1)$ [49]; sunflower seed (Helianthus annus) $(0.82 \%, n=1)$ [45]. In the case of tubers, only $4.55 \%(n=1)$ of the matrices represents this group, being the yam (Dioscorea spp.) that constituted $0.82 \%$ $(n=1)$ of beverages [41].

Regarding the origin of the 122 beverages and how the nutritional data were obtained, $54.92 \%(n=67)$ of the beverages were prepared for the study, of which $50.82 \%(n=62)$ were analyzed to determine their nutritional composition [31-40,42-51,55,56,58-60]; 3.28\% $(n=4)$ had their nutritional values determined based on the label of the product of origin [30]; $0.82 \%(n=1)$ had their nutritional data taken from Brazilian Nutritional Composition Table 
and Tables of Nutritional Composition of Foods Consumed in Brazil of IBGE (Instituto Brasileiro de Geografia e Estatística) [41,61,62].

Considering other beverages, $32.79 \%(n=40)$ were commercial plant-based beverages used as alternatives for cow's milk from different brands whose nutritional values were obtained from the labels [52-54,57]; $12.30 \%(n=15)$ had their nutritional composition evaluated using USDA (United States Department of Agriculture) Food Composition Database [23].

Table 1 shows the nutritional composition, ingredients and specifications on the origin of the beverages. The identification information (authors, year of publication and country of origin) collected from the studies is presented in Appendix B.

Considering Table 1, the energy value ranged from 6 to $183 \mathrm{Kcal} / 100 \mathrm{~mL}$, the carbohydrate content ranged from 0.00 to $22.29 \mathrm{~g} / 100 \mathrm{~mL}$, the protein content ranged from 0.06 to $12.43 \mathrm{~g} / 100 \mathrm{~mL}$, and the lipid content ranged from 0.00 to $19.00 \mathrm{~g} / 100 \mathrm{~mL}$. The most caloric beverage collected and with the highest lipid content is the coconut-based beverage from the Real Thai brand [54]. Four rice-based beverages of different types (parboiled, brown, polished and red) presented the lowest lipid value [30].

The least caloric and with the lowest protein content is the tucumã almond-based beverage extracted at a temperature of $55^{\circ} \mathrm{C}$ [35]. The beverage collected with the highest protein content is the concentrated fluid from stage 5 of the crioconcentration process that the sapucaia nut-based beverage was submitted to [47].

Regarding carbohydrates, the sesame-based beverage that contains maltodextrin as an ingredient has the highest carbohydrate content [34], while the sunflower seed-based has the lowest carbohydrate content [45]. Among the beverages collected that dietary fiber values were provided for $(18.03 \%, n=22)$, this nutrient content ranged from 0.00 to $4.40 \mathrm{~g} / 100 \mathrm{~mL}$. An oat-based beverage has the highest dietary fiber content [50], while three rice-based beverages of parboiled, polished and red rice types [30], and a coconut-based beverage [52] have the lowest dietary fiber value.

With regard to the collected micronutrients, the amount of beverages that provided these data were $44.26 \%(n=54)$ for calcium, $6.56 \%(n=8)$ for iron, $13.11 \%(n=16)$ for magnesium, and $22.95 \%(n=28)$ for sodium. The calcium content ranged from 0.00 to $1252.94 \mathrm{mg} / 100 \mathrm{~mL}$, iron content ranged from 0.04 to $1.40 \mathrm{mg} / 100 \mathrm{~mL}$, magnesium content ranged from 0.84 to $10,178.60 \mathrm{mg} / 100 \mathrm{~mL}$, and sodium content ranged from 0.00 to $343.43 \mathrm{mg} / 100 \mathrm{~mL}$.

The concentrated fluid from stage 5 of the crioconcentration process that the sapucaia nut-based beverage was submitted has a higher content of calcium, magnesium, and sodium [47]. An almond-based beverage has the highest content of iron [58].

Three rice-based beverages (with brown, parboiled and red rice types) [30], one soybased beverage and two coconut-based beverages [23] have the lowest calcium content. A rice-based beverage made with broken polished rice has the lowest content of iron and magnesium [46]. Considering sodium, three rice-based beverages (with brown, polished and red rice types) presented the lowest sodium content [30].

Excluding water and the matrix from the beverages, the most commonly found ingredients in plant-based beverages were salt (50 times), sugar (21 times), vitamin E (tocopherol) (20 times), tricalcium phosphate (18 times) and gellan gum (17 times) (Figure 1). 


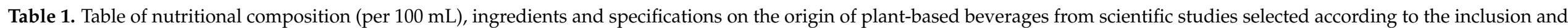
exclusion criteria.

\begin{tabular}{|c|c|c|c|c|c|c|c|c|c|c|c|}
\hline Authors and Year & Ingredients & $\begin{array}{l}\text { Energy } \\
\text { (Kcal) }\end{array}$ & $\begin{array}{c}\mathrm{CHO} \\
(\mathrm{g})\end{array}$ & $\begin{array}{l}\text { Protein } \\
\text { (g) }\end{array}$ & $\begin{array}{l}\text { Lipid } \\
\text { (g) }\end{array}$ & $\begin{array}{l}\text { Dietary } \\
\text { Fiber (g) }\end{array}$ & $\begin{array}{c}\mathrm{Ca} \\
(\mathrm{mg})\end{array}$ & $\begin{array}{c}\mathrm{Fe} \\
(\mathrm{mg})\end{array}$ & $\begin{array}{c}\mathrm{Mg} \\
(\mathrm{mg})\end{array}$ & $\begin{array}{l}\mathrm{Na} \\
(\mathrm{mg})\end{array}$ & $\begin{array}{l}\text { Specifications on the } \\
\text { Origin of the Beverage/ } \\
\text { Where the Nutritional } \\
\text { Data Were Obtained }\end{array}$ \\
\hline \multicolumn{12}{|c|}{ Almond-based beverage } \\
\hline $\begin{array}{l}\text { Alozie and Udofia, } \\
2015\end{array}$ & $\begin{array}{l}\text { Water, almond (dehulled), sugar syrup (granulated } \\
\text { sugar + water), vanilla essence. }\end{array}$ & 55 & 4.50 & 1.70 & 3.40 & 1.25 & 13.10 & 1.40 & 42.05 & 6.38 & $\begin{array}{l}\text { Beverage prepared and } \\
\text { analyzed for the study. }\end{array}$ \\
\hline $\begin{array}{l}\text { Chalupa-Krebzdak } \\
\text { et al., } 2018\end{array}$ & $\begin{array}{l}\text { Almond milk (filtered water, almonds), evaporated } \\
\text { cane juice syrup, calcium carbonate, sea salt, } \\
\text { potassium citrate, carrageenan, sunflower lecithin, } \\
\text { vitamin A palmitate, vitamin D2, D-alpha } \\
\text { tocopherol (natural vitamin E). }\end{array}$ & 25 & 3.33 & 0.42 & 1.04 & - & 188.00 & - & - & - & $\begin{array}{l}\text { USDA Food Composition } \\
\text { Database. }\end{array}$ \\
\hline $\begin{array}{l}\text { Chalupa-Krebzdak } \\
\text { et al., } 2018\end{array}$ & $\begin{array}{l}\text { Almond milk (filtered water, almonds), calcium } \\
\text { carbonate, tapioca starch, sea salt, potassium citrate, } \\
\text { carrageenan, sunflower lecithin, natural flavour, } \\
\text { vitamin A palmitate, vitamin D2 and D-alpha } \\
\text { tocopherol (natural vitamin E). }\end{array}$ & 12 & 0.62 & 0.31 & 1.08 & - & 185.00 & - & - & - & $\begin{array}{l}\text { USDA Food Composition } \\
\text { Database. }\end{array}$ \\
\hline $\begin{array}{l}\text { Chalupa-Krebzdak } \\
\text { et al., } 2018\end{array}$ & $\begin{array}{c}\text { Almond milk (filtered water, almonds), honey, cane } \\
\text { sugar, calcium carbonate, sea salt, potassium citrate, } \\
\text { carrageenan, sunflower lecithin, guar gum, natural } \\
\text { flavor, vitamin A palmitate, vitamin D2, D-alpha } \\
\text { tocopherol (natural vitamin E). }\end{array}$ & 21 & 3.33 & 0.42 & 1.04 & - & 188.00 & - & - & - & $\begin{array}{l}\text { USDA Food Composition } \\
\text { Database. }\end{array}$ \\
\hline $\begin{array}{l}\text { Chalupa-Krebzdak } \\
\text { et al., } 2018\end{array}$ & $\begin{array}{c}\text { Almond milk (water, almonds), pea protein, rice } \\
\text { protein, calcium phosphate, magnesium phosphate, } \\
\text { carrageenan, natural flavor, locust bean gum, kosher } \\
\text { sea salt, vitamin A palmitate, vitamin D2 } \\
\text { L-selenomethionine (selenium), zinc oxide, folic } \\
\text { acid, vitamin b-12. }\end{array}$ & 17 & 0.42 & 2.08 & 0.83 & - & 42.00 & - & - & - & $\begin{array}{l}\text { USDA Food Composition } \\
\text { Database. }\end{array}$ \\
\hline Decloedt et al., 2018 & $\begin{array}{l}\text { Water, almond ( } 2.10 \%) \text {, tricalcium phosphate, salt, } \\
\text { sunflower lecithine (emulsifier), sugar, locust bean } \\
\text { gum, gellan gum. }\end{array}$ & 24 & 3.00 & 0.50 & 1.10 & 0.20 & 120.00 & - & - & $56.00^{\mathrm{a}}$ & $\begin{array}{l}\text { Nutritional values } \\
\text { obtained from the label of } \\
\text { the drinks that were } \\
\text { purchased. }\end{array}$ \\
\hline Decloedt et al., 2018 & $\begin{array}{l}\text { Water, almond (2.10\%), tricalcium phosphate, salt, } \\
\text { sunflower lecithine (emulsifier), aromas, locust bean } \\
\text { gum, gellan gum. }\end{array}$ & 13 & 0.10 & 0.50 & 1.30 & 0.20 & 120.00 & - & - & $56.00^{\mathrm{a}}$ & $\begin{array}{l}\text { Nutritional values } \\
\text { obtained from the label of } \\
\text { the drinks that } \\
\text { were purchased. }\end{array}$ \\
\hline
\end{tabular}


Table 1. Cont.

\begin{tabular}{|c|c|c|c|c|c|c|c|c|c|c|c|}
\hline Authors and Year & Ingredients & $\begin{array}{l}\text { Energy } \\
\text { (Kcal) }\end{array}$ & $\begin{array}{c}\text { CHO } \\
\text { (g) }\end{array}$ & $\begin{array}{l}\text { Protein } \\
\text { (g) }\end{array}$ & $\begin{array}{l}\text { Lipid } \\
\text { (g) }\end{array}$ & $\begin{array}{l}\text { Dietary } \\
\text { Fiber (g) }\end{array}$ & $\begin{array}{c}\text { Ca } \\
(\mathrm{mg})\end{array}$ & $\begin{array}{c}\mathrm{Fe} \\
(\mathrm{mg})\end{array}$ & $\begin{array}{c}\mathrm{Mg} \\
(\mathrm{mg})\end{array}$ & $\begin{array}{c}\mathrm{Na} \\
(\mathrm{mg})\end{array}$ & $\begin{array}{l}\text { Specifications on the } \\
\text { Origin of the Beverage/ } \\
\text { Where the Nutritional } \\
\text { Data Were Obtained }\end{array}$ \\
\hline Jeske, 2018 & $\begin{array}{l}\text { Water, sugar, almond (2.00\%), tri-calcium phosphate, } \\
\text { sea salt, stabilizers (locust bean gum, gellan gum), } \\
\text { emulsifier (sunflower lecithin), vitamins B2, B12, E, D2). }\end{array}$ & $25^{\mathrm{b}}$ & $3.08^{b}$ & $0.51^{b}$ & $1.13^{b}$ & - & - & - & - & - & $\begin{array}{l}\text { Nutritional values } \\
\text { obtained from the label. } \\
\text { Brand name: Alpro. }\end{array}$ \\
\hline Jeske, 2018 & Water, almond $(7.00 \%)$, sea salt. & $34^{\mathrm{b}}$ & $0.21^{\mathrm{b}}$ & $0.92^{b}$ & $3.08^{b}$ & - & - & - & - & - & $\begin{array}{l}\text { Nutritional values } \\
\text { obtained from the label. } \\
\text { Brand name: Provamel. }\end{array}$ \\
\hline $\begin{array}{l}\text { Martínez-Padilla et al., } \\
\qquad 2020\end{array}$ & $\begin{array}{c}\text { Water, almond }(7.00 \%) \text {, tapioca starch, natural } \\
\text { almond flavoring. }\end{array}$ & 32 & 3.30 & 1.00 & 2.10 & - & - & - & - & - & $\begin{array}{l}\text { Nutritional values } \\
\text { obtained from the label. } \\
\text { Brand name: Ecomil. }\end{array}$ \\
\hline $\begin{array}{l}\text { Scholz-Ahrens et al., } \\
2020\end{array}$ & Water, almonds (6.50\%) sea salt. & $32^{b}$ & $0.21^{\mathrm{b}}$ & $0.82^{\mathrm{b}}$ & $2.98^{b}$ & - & ND & - & - & - & $\begin{array}{l}\text { Nutritional values } \\
\text { obtained from the label. } \\
\text { Brand name: Provamel. }\end{array}$ \\
\hline Silva, 2018 & Water, almonds. & $68^{\mathrm{b}}$ & $0.67^{\mathrm{b}}$ & $4.36^{\mathrm{b}}$ & $5.51^{\mathrm{b}}$ & $2.16^{\mathrm{b}}$ & - & - & - & - & $\begin{array}{l}\text { Beverage prepared and } \\
\text { analyzed for the study. }\end{array}$ \\
\hline \multicolumn{12}{|c|}{ Amaranth-based beverage } \\
\hline \multicolumn{12}{|c|}{ Baru almond-based beverage } \\
\hline Vieira, 2017 & Water, baru almond. & $71^{b}$ & $1.94^{\mathrm{b}}$ & $3.15^{b}$ & $5.66^{\mathrm{b}}$ & - & - & - & - & - & $\begin{array}{l}\text { Beverage prepared and } \\
\text { analyzed for the study. }\end{array}$ \\
\hline \multicolumn{12}{|c|}{ Cashew nut-based beverage } \\
\hline $\begin{array}{l}\text { Chalupa-Krebzdak } \\
\text { et al., } 2018\end{array}$ & $\begin{array}{l}\text { Cashew milk (filtered water, cashews), cane sugar, } \\
\text { tricalcium phosphate, sea salt, almond butter, locust } \\
\text { bean gum, sunflower lecithin, gellan gum, vitamin E } \\
\text { acetate, zinc gluconate, vitamin A palmitate, } \\
\text { riboflavin (B2) vitamin B12, vitamin D2. }\end{array}$ & 25 & 3.75 & 0.42 & 1.04 & - & 188.00 & - & - & - & $\begin{array}{l}\text { USDA Food Composition } \\
\text { Database. }\end{array}$ \\
\hline $\begin{array}{l}\text { Chalupa-Krebzdak } \\
\text { et al., } 2018\end{array}$ & $\begin{array}{l}\text { Cashew milk (filtered water, cashews), cane sugar, } \\
\text { tricalcium phosphate, sea salt, almond butter, locus } \\
\text { bean gum, sunflower lecithin, gellan gum, vitamin E } \\
\text { acetate, zinc gluconate, vitamin A palmitate, } \\
\text { riboflavin (B2) vitamin B12, vitamin D2. }\end{array}$ & 79 & 5.73 & 2.20 & 5.29 & - & 9.00 & - & - & - & $\begin{array}{l}\text { USDA Food Composition } \\
\text { Database. }\end{array}$ \\
\hline
\end{tabular}


Table 1. Cont.

\begin{tabular}{|c|c|c|c|c|c|c|c|c|c|c|c|}
\hline Authors and Year & Ingredients & $\begin{array}{l}\text { Energy } \\
\text { (Kcal) }\end{array}$ & $\begin{array}{c}\mathrm{CHO} \\
(\mathrm{g})\end{array}$ & $\begin{array}{l}\text { Protein } \\
\quad(g)\end{array}$ & $\begin{array}{l}\text { Lipid } \\
\text { (g) }\end{array}$ & $\begin{array}{l}\text { Dietary } \\
\text { Fiber (g) }\end{array}$ & $\begin{array}{l}\mathrm{Ca} \\
(\mathrm{mg})\end{array}$ & $\begin{array}{c}\mathrm{Fe} \\
(\mathrm{mg})\end{array}$ & $\begin{array}{l}\mathrm{Mg} \\
(\mathrm{mg})\end{array}$ & $\begin{array}{l}\mathrm{Na} \\
(\mathrm{mg})\end{array}$ & $\begin{array}{l}\text { Specifications on the } \\
\text { Origin of the Beverage/ } \\
\text { Where the Nutritional } \\
\text { Data Were Obtained }\end{array}$ \\
\hline Decloedt et al., 2018 & $\begin{array}{l}\text { Water, cashew nuts ( } 3.10 \%) \text {, tricalcium phosphate, } \\
\text { salt, sunflower lecithine (emulsifier), sugar, locust } \\
\text { bean gum, gellan gum. }\end{array}$ & 23 & 2.60 & 0.50 & 1.10 & 0.20 & 120.00 & - & - & $52.00^{\mathrm{a}}$ & $\begin{array}{l}\text { Nutritional values } \\
\text { obtained from the label of } \\
\text { the drinks that were } \\
\text { purchased. }\end{array}$ \\
\hline Holanda, 2017 & $\begin{array}{l}\text { Water, cashew nut, sugar, tricalcium } \\
\text { calcium phosphate. }\end{array}$ & $58^{b}$ & $4.92^{b}$ & $2.13^{b}$ & $3.29^{b}$ & - & $\begin{array}{c}108.76 \\
b\end{array}$ & - & - & - & $\begin{array}{l}\text { Beverage prepared and } \\
\text { analyzed for the study. }\end{array}$ \\
\hline Jeske, 2018 & $\begin{array}{l}\text { Water, roasted cashew }(6.00 \%) \text {, agave syrup }(3.50 \%) \text {, } \\
\text { sea salt. }\end{array}$ & $48^{\mathrm{b}}$ & $4.52^{\mathrm{b}}$ & $0.92^{b}$ & $2.88^{\mathrm{b}}$ & - & - & - & - & - & $\begin{array}{l}\text { Nutritional values } \\
\text { obtained from the label. } \\
\text { Brand name: Provamel. }\end{array}$ \\
\hline Lima et al., 2020 & Water, broken cashew nut kernels, sugar. & $66^{\mathrm{b}}$ & $5.58^{\mathrm{b}}$ & $1.88^{\mathrm{b}}$ & $4.08^{\mathrm{b}}$ & - & - & - & - & - & $\begin{array}{l}\text { Beverage prepared and } \\
\text { analyzed for the study. }\end{array}$ \\
\hline Manzoor et al., 2017 & Water, cashew nuts, sugar syrup, vanilla flavor. & $57^{b}$ & $4.50^{\mathrm{b}}$ & $2.11^{\mathrm{b}}$ & $3.39^{b}$ & $1.18^{\mathrm{b}}$ & 21.90 & 0.80 & 38.20 & 22.80 & $\begin{array}{l}\text { Beverage prepared and } \\
\text { analyzed for the study. }\end{array}$ \\
\hline $\begin{array}{l}\text { Scholz-Ahrens et al., } \\
2020\end{array}$ & $\begin{array}{l}\text { Water, cashew nuts (3.10\%), sugar, calcium } \\
\text { (tri-calcium phosphate), sea salt, stabilizers (locust } \\
\text { bean gum, gellan gum), emulsifier (sunflower } \\
\text { lecithin), vitamins (riboflavin (B2), B12, E, D2). }\end{array}$ & $24^{b}$ & $2.67^{b}$ & $0.51^{b}$ & $1.13^{\mathrm{b}}$ & - & ND & - & - & - & $\begin{array}{l}\text { Nutritional values } \\
\text { obtained from the label. } \\
\text { Brand name: Alpro. }\end{array}$ \\
\hline \multicolumn{12}{|c|}{ Coconut-based beverage } \\
\hline $\begin{array}{l}\text { Chalupa-Krebzdak } \\
\text { et al., } 2018\end{array}$ & $\begin{array}{l}\text { Organic coconut milk (or water, organic coconut } \\
\text { cream), organic dried cane syrup, chicory root } \\
\text { extract (inulin), tapioca dextrose, pectin, algin (kelp } \\
\text { extract), magnesium phosphate, tricalcium } \\
\text { phosphate, rice starch, natural flavours, locust bean } \\
\text { gum, live cultures, carrageenan, guar gum, } \\
\text { dipotassium phosphate, vitamin B12. }\end{array}$ & 76 & 9.41 & 0.59 & 4.12 & - & 176.00 & - & - & - & $\begin{array}{l}\text { USDA Food Composition } \\
\text { Database. }\end{array}$ \\
\hline $\begin{array}{l}\text { Chalupa-Krebzdak } \\
\text { et al., } 2018\end{array}$ & $\begin{array}{l}\text { Coconut extract }(25.00 \%) \text {, water, carboxymethyl } \\
\text { cellulose (E466, guar gum. }\end{array}$ & 92 & 7.00 & 2.00 & 6.00 & - & 0.00 & - & - & - & $\begin{array}{l}\text { USDA Food Composition } \\
\text { Database. }\end{array}$ \\
\hline $\begin{array}{l}\text { Chalupa-Krebzdak } \\
\text { et al., } 2018\end{array}$ & $\begin{array}{l}\text { Coconut milk, water, stabilizer, } \\
\text { sodium metabisulphite. }\end{array}$ & 50 & 3.75 & 1.25 & 5.00 & - & 0.00 & - & - & - & $\begin{array}{l}\text { USDA Food Composition } \\
\text { Database. }\end{array}$ \\
\hline Decloedt et al., 2018 & $\begin{array}{l}\text { Water, coconut milk (coconut cream and water) } \\
(5.30 \%) \text {, rice }(3.30 \%) \text {, tricalcium phosphate, salt (sea), } \\
\text { aromas, carrageenan, guar gum, xanthan gum. }\end{array}$ & 20 & 2.70 & 0.10 & 0.90 & 0.00 & 120.00 & - & - & $52.00^{\mathrm{a}}$ & $\begin{array}{l}\text { Nutritional values } \\
\text { obtained from the label of } \\
\text { the drinks that } \\
\text { were purchased. }\end{array}$ \\
\hline
\end{tabular}


Table 1. Cont.

\begin{tabular}{|c|c|c|c|c|c|c|c|c|c|c|c|}
\hline Authors and Year & Ingredients & $\begin{array}{c}\text { Energy } \\
\text { (Kcal) }\end{array}$ & $\underset{\text { (g) }}{\mathrm{CHO}}$ & $\begin{array}{l}\text { Protein } \\
\text { (g) }\end{array}$ & $\begin{array}{l}\text { Lipid } \\
\text { (g) }\end{array}$ & $\begin{array}{l}\text { Dietary } \\
\text { Fiber (g) }\end{array}$ & $\begin{array}{c}\mathrm{Ca} \\
(\mathrm{mg})\end{array}$ & $\begin{array}{c}\mathrm{Fe} \\
(\mathrm{mg})\end{array}$ & $\begin{array}{l}\mathrm{Mg} \\
(\mathrm{mg})\end{array}$ & $\begin{array}{c}\mathrm{Na} \\
(\mathrm{mg})\end{array}$ & $\begin{array}{l}\text { Specifications on the } \\
\text { Origin of the Beverage/ } \\
\text { Where the Nutritional } \\
\text { Data Were Obtained }\end{array}$ \\
\hline Jeske, 2018 & $\begin{array}{c}\text { Water, coconut milk (5.30\%) (coconut cream, water), } \\
\text { rice }(3.30 \%) \text {, tri-calcium phosphate, stabilizers } \\
\text { (carrageenan, guar gum, Xanthan gum), sea salt, } \\
\text { vitamins (B12, D2), flavorings. }\end{array}$ & $21^{\mathrm{b}}$ & $2.77^{\mathrm{b}}$ & $0.10^{\mathrm{b}}$ & $0.92^{\mathrm{b}}$ & - & - & - & - & - & $\begin{array}{l}\text { Nutritional values } \\
\text { obtained from the label. } \\
\text { Brand name: Alpro. }\end{array}$ \\
\hline $\begin{array}{l}\text { Martínez-Padilla et al., } \\
2020\end{array}$ & $\begin{array}{l}\text { Water, coconut milk ( } 5.30 \% \text { ), raw cane sugar, } \\
\text { maltodextrin, algae Lithothamnium calcareum. }\end{array}$ & 26 & 4.10 & 0.10 & 0.90 & - & - & - & - & - & $\begin{array}{l}\text { Nutritional values } \\
\text { obtained from the label. } \\
\text { Brand name: Naturli. }\end{array}$ \\
\hline $\begin{array}{l}\text { Scholz-Ahrens et al., } \\
2020\end{array}$ & Coconut extract $85.00 \%$, water. & $183^{\mathrm{b}}$ & $2.05^{\mathrm{b}}$ & $1.64^{\mathrm{b}}$ & $19.00^{\mathrm{b}}$ & - & ND & - & - & - & $\begin{array}{l}\text { Nutritional values } \\
\text { obtained from the label. } \\
\text { Brand name: Real Thai. }\end{array}$ \\
\hline $\begin{array}{l}\text { Scholz-Ahrens et al., } \\
2020\end{array}$ & Coconut milk (30.00\%), water, corn starch. & $46^{\mathrm{b}}$ & $0.51^{\mathrm{b}}$ & $0.51^{\mathrm{b}}$ & $4.62^{\mathrm{b}}$ & - & ND & - & - & - & $\begin{array}{l}\text { Nutritional values } \\
\text { obtained from the label. } \\
\text { Brand name: Renuka. }\end{array}$ \\
\hline \multicolumn{12}{|c|}{ Cupuaçu almond-based beverage } \\
\hline Silva et al., 2015 & Water, cupuaçu almond flour. & $7^{b}$ & $0.26^{\mathrm{b}}$ & $0.10^{\mathrm{b}}$ & $0.63^{\mathrm{b}}$ & - & - & - & - & - & $\begin{array}{l}\text { Beverage prepared } \\
\text { (extraction temperature of } \\
55^{\circ} \mathrm{C} \text { ) and analyzed for } \\
\text { the study. }\end{array}$ \\
\hline Silva et al., 2015 & Water, cupuaçu almond flour. & $9^{b}$ & $0.46^{\mathrm{b}}$ & $0.07^{\mathrm{b}}$ & $0.81^{\mathrm{b}}$ & - & - & - & - & - & $\begin{array}{l}\text { Beverage prepared } \\
\text { (extraction temperature of } \\
75^{\circ} \mathrm{C} \text { ) and analyzed for } \\
\text { the study. }\end{array}$ \\
\hline Silva et al., 2015 & Water, cupuaçu almond flour. & $10^{\mathrm{b}}$ & $0.77^{\mathrm{b}}$ & $0.13^{\mathrm{b}}$ & $0.75^{\mathrm{b}}$ & - & - & - & - & - & $\begin{array}{l}\text { Beverage prepared } \\
\text { (extraction temperature of } \\
100^{\circ} \mathrm{C} \text { ) and analyzed for } \\
\text { the study. }\end{array}$ \\
\hline \multicolumn{12}{|c|}{ Groundnut-based beverage } \\
\hline Meeshi et al., 2014 & $\begin{array}{l}\text { Water, groundnut, sodium bicarbonate } \\
\text { (1.00\% solution). }\end{array}$ & 72 & 4.13 & 3.10 & 4.80 & - & 33.47 & - & - & - & $\begin{array}{l}\text { Beverage prepared and } \\
\text { analyzed for the study. }\end{array}$ \\
\hline
\end{tabular}


Table 1. Cont.

\begin{tabular}{|c|c|c|c|c|c|c|c|c|c|c|c|}
\hline Authors and Year & Ingredients & $\begin{array}{c}\text { Energy } \\
\text { (Kcal) }\end{array}$ & $\underset{(g)}{\mathrm{CHO}}$ & $\begin{array}{l}\text { Protein } \\
\text { (g) }\end{array}$ & $\begin{array}{l}\text { Lipid } \\
\text { (g) }\end{array}$ & $\begin{array}{l}\text { Dietary } \\
\text { Fiber (g) }\end{array}$ & $\begin{array}{c}\mathrm{Ca} \\
(\mathrm{mg})\end{array}$ & $\begin{array}{c}\mathrm{Fe} \\
(\mathrm{mg})\end{array}$ & $\begin{array}{c}\mathrm{Mg} \\
(\mathrm{mg})\end{array}$ & $\begin{array}{c}\mathrm{Na} \\
(\mathrm{mg})\end{array}$ & $\begin{array}{l}\text { Specifications on the } \\
\text { Origin of the Beverage/ } \\
\text { Where the Nutritional } \\
\text { Data Were Obtained }\end{array}$ \\
\hline \multicolumn{12}{|c|}{ Hazelnut-based beverage } \\
\hline Jeske, 2018 & $\begin{array}{c}\text { Water, sugar, hazelnuts (2.50\%), tri-calcium } \\
\text { phosphate, sea salt, stabilizers (locust bean gum, } \\
\text { gellan gum), emulsifier (sunflower lecithin), } \\
\text { vitamins B2, B12, E, D2). }\end{array}$ & $30^{\mathrm{b}}$ & $3.18^{\mathrm{b}}$ & $0.41^{\mathrm{b}}$ & $1.64^{\mathrm{b}}$ & - & - & - & - & - & $\begin{array}{l}\text { Nutritional values } \\
\text { obtained from the label. } \\
\text { Brand name: Alpro. }\end{array}$ \\
\hline $\begin{array}{l}\text { Martínez-Padilla et al., } \\
2020\end{array}$ & $\begin{array}{c}\text { Water, sugar, hazelnuts (2.50\%), calcium (tri-calcium } \\
\text { phosphate), sea salt, stabilizers (locust bean gum, } \\
\text { gellan gum), emulsifier (sunflower lecithin), } \\
\text { vitamins (riboflavin B2, B12, E, D2). }\end{array}$ & 29 & 3.10 & 0.40 & 1.60 & - & - & - & - & - & $\begin{array}{l}\text { Nutritional values } \\
\text { obtained from the label. } \\
\text { Brand name: Alpro. }\end{array}$ \\
\hline $\begin{array}{l}\text { Scholz-Ahrens et al., } \\
2020\end{array}$ & $\begin{array}{l}\text { Water, sugar, hazelnuts (2.50\%), calcium (tri-calcium } \\
\text { phosphate), sea salt, stabilizers (locust bean gum, } \\
\text { gellan gum), emulsifier (sunflower lecithin), } \\
\text { vitamins (riboflavin (B2), B12, E, D2). }\end{array}$ & $30^{\mathrm{b}}$ & $3.18^{\mathrm{b}}$ & $0.41^{\mathrm{b}}$ & $1.64^{\mathrm{b}}$ & - & $\underset{\mathrm{b}}{123.24}$ & - & - & - & $\begin{array}{l}\text { Nutritional values } \\
\text { obtained from the label. } \\
\text { Brand name: Alpro. }\end{array}$ \\
\hline $\begin{array}{l}\text { Scholz-Ahrens et al., } \\
2020\end{array}$ & $\begin{array}{l}\text { Water, European hazelnuts (4.00\%), agave syrup } \\
\qquad(3.50 \%) \text {, sea salt. }\end{array}$ & $37^{\mathrm{b}}$ & $2.67^{b}$ & $0.62^{\mathrm{b}}$ & $2.67^{\mathrm{b}}$ & - & ND & - & - & - & $\begin{array}{c}\text { Nutritional values } \\
\text { obtained from the label. } \\
\text { Brand name: Provamel. }\end{array}$ \\
\hline $\begin{array}{l}\text { Chalupa-Krebzdak } \\
\text { et al., } 2018\end{array}$ & $\begin{array}{c}\text { Organic flax/hemp cream (filtered water, organic } \\
\text { flax oil, organic hemp oil), organic brown rice solids, } \\
\text { organic brown rice syrup, organic tapioca, } \\
\text { non-GMO sunflower lecithin, Himalayan salt, } \\
\text { organic guar gum, xanthan gum. }\end{array}$ & 19 & 2.50 & 0.83 & 1.25 & - & 12.00 & - & - & - & $\begin{array}{l}\text { USDA Food Composition } \\
\text { Database. }\end{array}$ \\
\hline Jeske, 2018 & $\begin{array}{c}\text { Water, hemp cream (3.00\%), tri-calcium phosphate, } \\
\text { emulsifier (sucrose ester), natural flavoring, } \\
\text { stabilizer (xanthan gum), sea salt, stabilizer (gellan } \\
\text { gum), vitamin D2. }\end{array}$ & $24^{\mathrm{b}}$ & $0.10^{\mathrm{b}}$ & $0.10^{\mathrm{b}}$ & $2.77^{\mathrm{b}}$ & - & - & - & - & - & $\begin{array}{l}\text { Nutritional values } \\
\text { obtained from the label. } \\
\text { Brand name: Braham } \\
\text { and Murray. }\end{array}$ \\
\hline $\begin{array}{l}\text { Martínez-Padilla et al., } \\
2020\end{array}$ & $\begin{array}{l}\text { Water, hemp seeds ( } 3.00 \%) \text {, hemp oil }(1.30 \%) \text {, } \\
\text { tapioca starch, emulsifier: sunflower lecithin. }\end{array}$ & 40 & 2.20 & 1.00 & 2.90 & - & - & - & - & - & $\begin{array}{l}\text { Nutritional values } \\
\text { obtained from the label. } \\
\text { Brand name: Ecomil. }\end{array}$ \\
\hline
\end{tabular}


Table 1. Cont.

\begin{tabular}{|c|c|c|c|c|c|c|c|c|c|c|c|}
\hline Authors and Year & Ingredients & $\begin{array}{l}\text { Energy } \\
\text { (Kcal) }\end{array}$ & $\begin{array}{c}\mathrm{CHO} \\
(\mathrm{g})\end{array}$ & $\begin{array}{l}\text { Protein } \\
\quad(g)\end{array}$ & $\begin{array}{l}\text { Lipid } \\
\text { (g) }\end{array}$ & $\begin{array}{l}\text { Dietary } \\
\text { Fiber }(\mathrm{g})\end{array}$ & $\begin{array}{c}\mathrm{Ca} \\
(\mathrm{mg})\end{array}$ & $\begin{array}{c}\mathrm{Fe} \\
(\mathrm{mg})\end{array}$ & $\begin{array}{l}\mathrm{Mg} \\
(\mathrm{mg})\end{array}$ & $\begin{array}{l}\mathrm{Na} \\
(\mathrm{mg})\end{array}$ & $\begin{array}{l}\text { Specifications on the } \\
\text { Origin of the Beverage/ } \\
\text { Where the Nutritional } \\
\text { Data Were Obtained }\end{array}$ \\
\hline \multicolumn{12}{|c|}{ Macadamia nut-based beverage } \\
\hline Jeske, 2018 & $\begin{array}{l}\text { Water, macadamia nuts ( } 4.00 \%) \text {, agave syrup } \\
(3.50 \%) \text {, sea salt. }\end{array}$ & $35^{\mathrm{b}}$ & $2.46^{\mathrm{b}}$ & $0.51^{\mathrm{b}}$ & $2.46^{\mathrm{b}}$ & - & - & - & - & - & $\begin{array}{l}\text { Nutritional values } \\
\text { obtained from the label. } \\
\text { Brand name: Provamel. }\end{array}$ \\
\hline $\begin{array}{l}\text { Scholz-Ahrens et al., } \\
2020\end{array}$ & $\begin{array}{l}\text { Water, macadamia nuts ( } 4.00 \%) \text {, agave syrup } \\
(3.50 \%) \text {, sea salt. }\end{array}$ & $38^{\mathrm{b}}$ & $2.77^{b}$ & $0.31^{\mathrm{b}}$ & $2.67^{b}$ & - & ND & - & - & - & $\begin{array}{l}\text { Nutritional values } \\
\text { obtained from the label. } \\
\text { Brand name: Provamel. }\end{array}$ \\
\hline \multicolumn{12}{|c|}{ Millet-based beverage } \\
\hline $\begin{array}{l}\text { Scholz-Ahrens et al., } \\
2020\end{array}$ & $\begin{array}{c}\text { Water, millet }(12.00 \%) \text {, high-oleic sunflower oil, } \\
\text { sea salt. }\end{array}$ & $51^{b}$ & $9.24^{\mathrm{b}}$ & $0.51^{\mathrm{b}}$ & $1.54^{\mathrm{b}}$ & - & ND & - & - & - & $\begin{array}{l}\text { Nutritional values } \\
\text { obtained from the label. } \\
\text { Brand name: Swiss } \\
\text { cereal drink. }\end{array}$ \\
\hline \multicolumn{12}{|c|}{ Oat-based beverage } \\
\hline Andrade, 2018 & Water, oats. & $35^{\mathrm{b}}$ & $1.00^{\mathrm{b}}$ & $1.86^{\mathrm{b}}$ & $2.69^{b}$ & $3.21^{\mathrm{b}}$ & - & - & - & - & $\begin{array}{l}\text { Beverage prepared and } \\
\text { analyzed for the study. }\end{array}$ \\
\hline Decloedt et al., 2018 & $\begin{array}{l}\text { Water, oats }(10.00 \%) \text {, canola oil, calcium carbonate, } \\
\text { tricalcium phosphate (and other), salt. }\end{array}$ & 45 & 6.50 & 1.00 & 1.50 & 0.80 & 120.00 & - & - & $40.00^{\mathrm{a}}$ & $\begin{array}{l}\text { Nutritional values } \\
\text { obtained from the label of } \\
\text { the drinks that were } \\
\text { purchased. }\end{array}$ \\
\hline Jeske, 2018 & Oat base (water, oats $10.00 \%$ ), sea salt. & $36^{\mathrm{b}}$ & $6.68^{\mathrm{b}}$ & $1.03^{\mathrm{b}}$ & $0.51^{\mathrm{b}}$ & - & - & - & - & - & $\begin{array}{l}\text { Nutritional values } \\
\text { obtained from the label. } \\
\text { Brand name: Oatly. }\end{array}$ \\
\hline $\begin{array}{l}\text { Martínez-Padilla et al., } \\
\quad 2020\end{array}$ & Water, oats $(10.00 \%)$, sea salt. & 36 & 6.50 & 1.00 & 0.50 & - & - & - & - & - & $\begin{array}{c}\text { Nutritional values } \\
\text { obtained from the label. } \\
\text { Brand name: } \\
\text { Oatly organic. }\end{array}$ \\
\hline $\begin{array}{l}\text { Ravindran and } \\
\text { RadhaiSri, } 2020\end{array}$ & Water, oats. & 33 & 7.30 & 0.89 & 0.37 & 4.40 & - & - & - & - & $\begin{array}{l}\text { Beverage prepared and } \\
\text { analyzed for the study. }\end{array}$ \\
\hline
\end{tabular}


Table 1. Cont.

\begin{tabular}{|c|c|c|c|c|c|c|c|c|c|c|c|}
\hline Authors and Year & Ingredients & $\begin{array}{l}\text { Energy } \\
\text { (Kcal) }\end{array}$ & $\begin{array}{c}\mathrm{CHO} \\
(\mathrm{g})\end{array}$ & $\begin{array}{l}\text { Protein } \\
\quad(g)\end{array}$ & $\begin{array}{l}\text { Lipid } \\
\text { (g) }\end{array}$ & $\begin{array}{c}\text { Dietary } \\
\text { Fiber (g) }\end{array}$ & $\begin{array}{l}\mathrm{Ca} \\
(\mathrm{mg})\end{array}$ & $\begin{array}{c}\mathrm{Fe} \\
(\mathrm{mg})\end{array}$ & $\begin{array}{c}\mathrm{Mg} \\
(\mathrm{mg})\end{array}$ & $\begin{array}{c}\mathrm{Na} \\
(\mathrm{mg})\end{array}$ & $\begin{array}{l}\text { Specifications on the } \\
\text { Origin of the Beverage/ } \\
\text { Where the Nutritional } \\
\text { Data Were Obtained }\end{array}$ \\
\hline $\begin{array}{l}\text { Scholz-Ahrens et al., } \\
2020\end{array}$ & $\begin{array}{l}\text { Water, oat }(10.00 \%) \text {, rapeseed oil, algae } \\
\text { (Lithothamnium calcareum), sea salt, citric acid. }\end{array}$ & $46^{\mathrm{b}}$ & $6.68^{b}$ & $1.03^{\mathrm{b}}$ & $1.54^{\mathrm{b}}$ & - & $\underset{\mathrm{b}}{123.24}$ & - & - & - & $\begin{array}{l}\text { Nutritional values } \\
\text { obtained from the label. } \\
\text { Brand name: Oatly. }\end{array}$ \\
\hline \multicolumn{12}{|c|}{ Quinoa-based beverage } \\
\hline Jeske, 2018 & $\begin{array}{l}\text { Water, quinoa }(7.00 \%) \text {, agave syrup, corn } \\
\text { maltodextrin, almond oil. }\end{array}$ & $47^{\mathrm{b}}$ & $3.80^{\mathrm{b}}$ & $1.54^{\mathrm{b}}$ & $2.88^{\mathrm{b}}$ & - & - & - & - & - & $\begin{array}{l}\text { Nutritional values } \\
\text { obtained from the label. } \\
\text { Brand name: EcoMil. }\end{array}$ \\
\hline $\begin{array}{l}\text { Martínez-Padilla et al., } \\
\qquad 2020\end{array}$ & $\begin{array}{l}\text { Water, quinoa (4.00\%), inulin (agave fiber), } \\
\text { sunflower oil, emulsifier: sunflower lecithin. }\end{array}$ & 29 & 3.50 & 0.50 & 1.20 & - & - & - & - & - & $\begin{array}{l}\text { Nutritional values } \\
\text { obtained from the label. } \\
\text { Brand name: Ecomil. }\end{array}$ \\
\hline Vieira, 2013 & $\begin{array}{l}\text { Water (distilled), quinoa, saline solution } 0.03 \mathrm{M} \\
\text { (sodium chloride + distilled water), Termamyl enzyme, } \\
\text { amyloglucosidase enzyme, sunflower oil (1.00\%). }\end{array}$ & 35 & 5.47 & 1.02 & 1.04 & - & - & - & - & - & $\begin{array}{l}\text { Beverage prepared and } \\
\text { analyzed for the study. }\end{array}$ \\
\hline \multicolumn{12}{|c|}{ Rice-based beverage } \\
\hline Abrão, 2019 & Water, brown rice. & $20^{\mathrm{b}}$ & $4.20^{\mathrm{b}}$ & $0.40^{\mathrm{b}}$ & $0.00^{\mathrm{b}}$ & $0.35^{\mathrm{b}}$ & $0.00^{\mathrm{b}}$ & - & - & $0.00^{\mathrm{b}}$ & $\begin{array}{l}\text { Beverage prepared for the } \\
\text { study. Nutritional values } \\
\text { based on the label of the } \\
\text { product of origin. }\end{array}$ \\
\hline Abrão, 2019 & Water, parboiled rice. & $21^{b}$ & $4.60^{\mathrm{b}}$ & $0.40^{\mathrm{b}}$ & $0.00^{\mathrm{b}}$ & $0.00^{\mathrm{b}}$ & $0.00^{\mathrm{b}}$ & - & - & $0.65^{\mathrm{b}}$ & $\begin{array}{l}\text { Beverage prepared for the } \\
\text { study. Nutritional values } \\
\text { based on the label of the } \\
\text { product of origin. }\end{array}$ \\
\hline Abrão, 2019 & Water, polished rice. & $21^{\mathrm{b}}$ & $5.00^{\mathrm{b}}$ & $0.35^{b}$ & $0.00^{\mathrm{b}}$ & $0.00^{\mathrm{b}}$ & $2.50^{\mathrm{b}}$ & - & - & $0.00^{\mathrm{b}}$ & $\begin{array}{l}\text { Beverage prepared for the } \\
\text { study. Nutritional values } \\
\text { based on the label of the } \\
\text { product of origin. }\end{array}$ \\
\hline Abrão, 2019 & Water, red rice. & $21^{b}$ & $4.20^{\mathrm{b}}$ & $0.35^{b}$ & $0.00^{\mathrm{b}}$ & $0.00^{\mathrm{b}}$ & $0.00^{\mathrm{b}}$ & - & - & $0.00^{\mathrm{b}}$ & $\begin{array}{l}\text { Beverage prepared for the } \\
\text { study. Nutritional values } \\
\text { based on the label of the } \\
\text { product of origin. }\end{array}$ \\
\hline Carvalho et al., 2011 & $\begin{array}{l}\text { Water, broken polished rice of the EPAGRI } \\
109 \text { variety. }\end{array}$ & $18^{\mathrm{b}}$ & $3.25^{b}$ & $0.75^{b}$ & $0.42^{\mathrm{b}}$ & - & $0.90^{\mathrm{b}}$ & $0.04^{\mathrm{b}}$ & $0.84^{\mathrm{b}}$ & - & $\begin{array}{l}\text { Beverage prepared and } \\
\text { analyzed for the study. }\end{array}$ \\
\hline
\end{tabular}


Table 1. Cont.

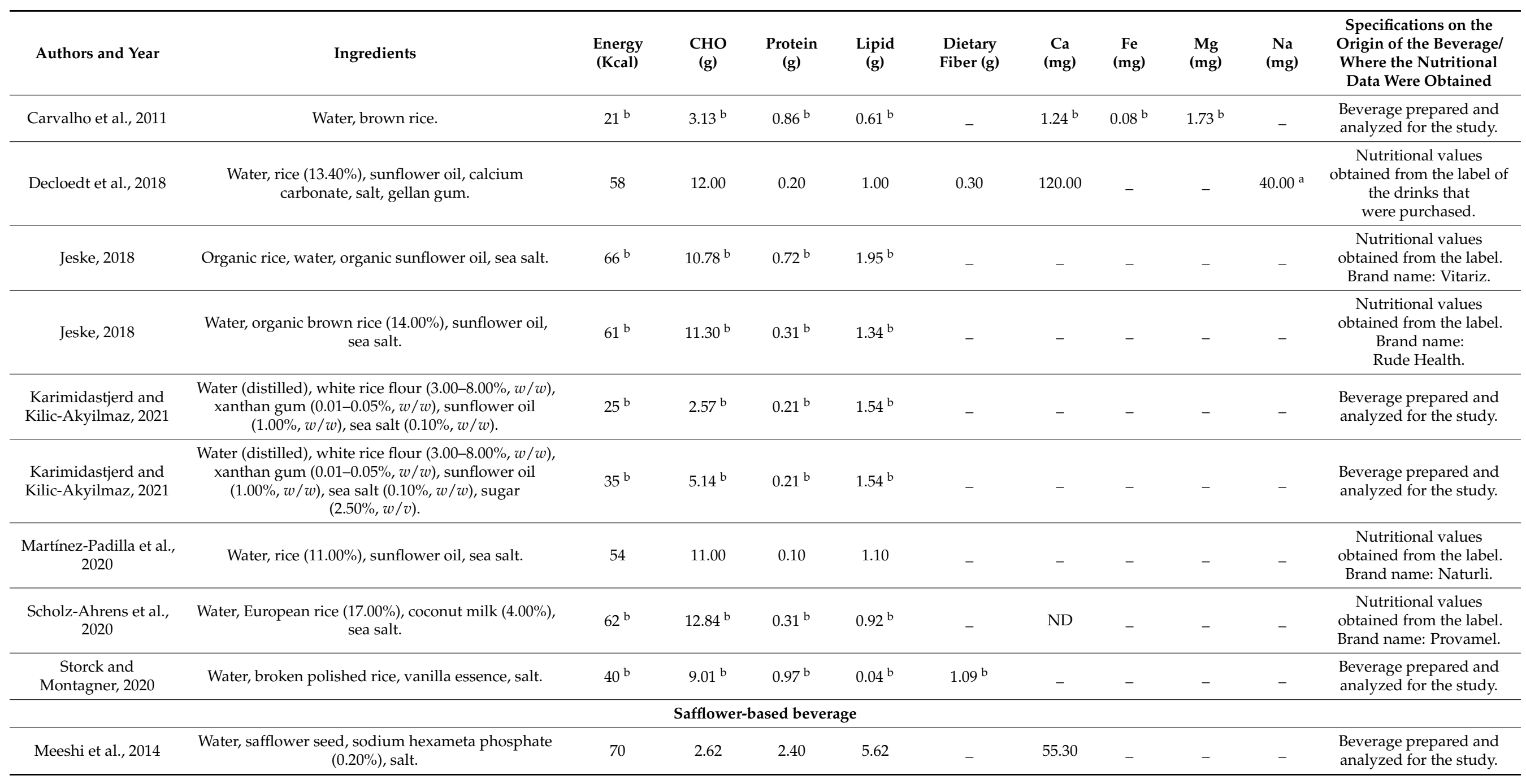


Table 1. Cont.

\begin{tabular}{|c|c|c|c|c|c|c|c|c|c|c|c|}
\hline Authors and Year & Ingredients & $\begin{array}{c}\text { Energy } \\
\text { (Kcal) }\end{array}$ & $\begin{array}{c}\mathrm{CHO} \\
(\mathrm{g})\end{array}$ & $\begin{array}{l}\text { Protein } \\
\text { (g) }\end{array}$ & $\begin{array}{l}\text { Lipid } \\
\text { (g) }\end{array}$ & $\begin{array}{c}\text { Dietary } \\
\text { Fiber (g) }\end{array}$ & $\begin{array}{c}\mathrm{Ca} \\
(\mathrm{mg})\end{array}$ & $\begin{array}{c}\mathrm{Fe} \\
(\mathrm{mg})\end{array}$ & $\begin{array}{c}\mathrm{Mg} \\
(\mathrm{mg})\end{array}$ & $\begin{array}{l}\mathrm{Na} \\
(\mathrm{mg})\end{array}$ & $\begin{array}{l}\text { Specifications on the } \\
\text { Origin of the Beverage/ } \\
\text { Where the Nutritional } \\
\text { Data Were Obtained }\end{array}$ \\
\hline \multicolumn{12}{|c|}{ Sapucaia nut-based beverage } \\
\hline Demoliner, 2019 & Water (distilled), sapucaia nut pie. & $54^{\mathrm{b}}$ & $1.34^{\mathrm{b}}$ & $1.94^{\mathrm{b}}$ & $4.51^{\mathrm{b}}$ & - & $\underset{\mathrm{b}}{636.74}$ & - & $\underset{\mathrm{b}}{2916.68}$ & $\underset{\mathrm{b}}{315.80}$ & $\begin{array}{l}\text { Beverage prepared } \\
\text { (submitted to the } \\
\text { crioconcentration } \\
\text { method-initial volume) } \\
\text { and analyzed for } \\
\text { the study. }\end{array}$ \\
\hline Demoliner, 2019 & Water (distilled), sapucaia nut pie. & $40^{\mathrm{b}}$ & $2.10^{\mathrm{b}}$ & $2.36^{\mathrm{b}}$ & $2.42^{\mathrm{b}}$ & - & $\underset{\mathrm{b}}{709.66}$ & - & $\underset{\mathrm{b}}{3183.70}$ & $\underset{\mathrm{b}}{318.78}$ & $\begin{array}{l}\text { Beverage prepared } \\
\text { (submitted to the } \\
\text { crioconcentration } \\
\text { method-concentrated } \\
\text { fluid 1) and analyzed for } \\
\text { the study. }\end{array}$ \\
\hline Demoliner, 2019 & Water (distilled), sapucaia nut pie. & $60^{\mathrm{b}}$ & $1.03^{\mathrm{b}}$ & $2.19^{\mathrm{b}}$ & $5.26^{\mathrm{b}}$ & - & $\underset{\mathrm{b}}{523.77}$ & - & $\underset{\mathrm{b}}{1008.51}$ & $\underset{\mathrm{b}}{120.06}$ & $\begin{array}{l}\text { Beverage prepared } \\
\text { (submitted to the } \\
\text { crioconcentration } \\
\text { method-ice 1) and } \\
\text { analyzed for the study. }\end{array}$ \\
\hline Demoliner, 2019 & Water (distilled), sapucaia nut pie. & $50^{\mathrm{b}}$ & $3.97^{b}$ & $2.67^{\mathrm{b}}$ & $2.55^{\mathrm{b}}$ & - & $\begin{array}{c}738.41 \\
\mathrm{~b}\end{array}$ & - & $\begin{array}{c}3382.94 \\
\mathrm{~b}\end{array}$ & $\underset{\mathrm{b}}{325.66}$ & $\begin{array}{l}\text { Beverage prepared } \\
\text { (submitted to the } \\
\text { crioconcentration } \\
\text { method-concentrated } \\
\text { fluid 2) and analyzed for } \\
\text { the study. }\end{array}$ \\
\hline Demoliner, 2019 & Water (distilled), sapucaia nut pie. & $45^{\mathrm{b}}$ & $2.67^{b}$ & $0.91^{\mathrm{b}}$ & $3.43^{b}$ & - & $\underset{\mathrm{b}}{517.61}$ & - & $\underset{\mathrm{b}}{1283.75}$ & $\underset{\mathrm{b}}{192.25}$ & $\begin{array}{l}\text { Beverage prepared } \\
\text { (submitted to the } \\
\text { crioconcentration } \\
\text { method-ice 2) and } \\
\text { analyzed for the study. }\end{array}$ \\
\hline Demoliner, 2019 & Water (distilled), sapucaia nut pie. & $56^{\mathrm{b}}$ & $4.29^{b}$ & $4.40^{\mathrm{b}}$ & $2.32^{b}$ & - & $\underset{\mathrm{b}}{862.99}$ & - & $\underset{\mathrm{b}}{4467.45}$ & $\underset{\mathrm{b}}{323.71}$ & $\begin{array}{l}\text { Beverage prepared } \\
\text { (submitted to the } \\
\text { crioconcentration } \\
\text { method-concentrated } \\
\text { fluid 3) and analyzed for } \\
\text { the study. }\end{array}$ \\
\hline
\end{tabular}


Table 1. Cont.

\begin{tabular}{|c|c|c|c|c|c|c|c|c|c|c|c|}
\hline Authors and Year & Ingredients & $\begin{array}{l}\text { Energy } \\
\text { (Kcal) }\end{array}$ & $\begin{array}{l}\text { CHO } \\
\text { (g) }\end{array}$ & $\begin{array}{l}\text { Protein } \\
\text { (g) }\end{array}$ & $\begin{array}{l}\text { Lipid } \\
\text { (g) }\end{array}$ & $\begin{array}{l}\text { Dietary } \\
\text { Fiber (g) }\end{array}$ & $\begin{array}{c}\mathrm{Ca} \\
(\mathrm{mg})\end{array}$ & $\begin{array}{c}\mathrm{Fe} \\
(\mathrm{mg})\end{array}$ & $\begin{array}{c}\mathrm{Mg} \\
(\mathrm{mg})\end{array}$ & $\begin{array}{l}\mathrm{Na} \\
(\mathrm{mg})\end{array}$ & $\begin{array}{l}\text { Specifications on the } \\
\text { Origin of the Beverage/ } \\
\text { Where the Nutritional } \\
\text { Data Were Obtained }\end{array}$ \\
\hline Demoliner, 2019 & Water (distilled), sapucaia nut pie. & $21^{b}$ & $2.75^{b}$ & $0.84^{\mathrm{b}}$ & $0.69^{b}$ & - & $\underset{\mathrm{b}}{515.55}$ & - & $\begin{array}{c}1345.37 \\
\mathrm{~b}\end{array}$ & $\underset{\mathrm{b}}{262.09}$ & $\begin{array}{l}\text { Beverage prepared } \\
\text { (submitted to the } \\
\text { crioconcentration } \\
\text { method-ice 3) and } \\
\text { analyzed for the study. }\end{array}$ \\
\hline Demoliner, 2019 & Water (distilled), sapucaia nut pie. & $11^{\mathrm{b}}$ & $3.19^{b}$ & $0.77^{\mathrm{b}}$ & $3.65^{\mathrm{b}}$ & - & $\underset{\mathrm{b}}{952.23}$ & - & $\underset{\mathrm{b}}{6264.70}$ & $\underset{\mathrm{b}}{163.50}$ & $\begin{array}{l}\text { Beverage prepared } \\
\text { (submitted to the } \\
\text { crioconcentration } \\
\text { method-concentrated } \\
\text { fluid 4) and analyzed for } \\
\text { the study. } \\
\text { Beverage prepared } \\
\text { (submitted to the } \\
\text { crioconcentration } \\
\text { method-ice 4) and } \\
\text { analyzed for the study. }\end{array}$ \\
\hline Demoliner, 2019 & Water (distilled), sapucaia nut pie. & $119^{\mathrm{b}}$ & $7.98^{\mathrm{b}}$ & $12.43^{b}$ & $4.12^{\mathrm{b}}$ & - & $\underset{\mathrm{b}}{1252.94}$ & - & $\begin{array}{c}10,178.60 \\
b\end{array}$ & $\underset{\mathrm{b}}{343.43}$ & $\begin{array}{l}\text { Beverage prepared } \\
\text { (submitted to the } \\
\text { crioconcentration } \\
\text { method-concentrated } \\
\text { fluid 5) and analyzed for } \\
\text { the study. }\end{array}$ \\
\hline Demoliner, 2019 & Water (distilled), sapucaia nut pie. & $22^{b}$ & $1.83^{\mathrm{b}}$ & $2.00^{\mathrm{b}}$ & $0.77^{\mathrm{b}}$ & - & $\underset{\mathrm{b}}{752.79}$ & - & $\underset{b}{1663.74}$ & $\underset{\mathrm{b}}{292.90}$ & $\begin{array}{l}\text { Beverage prepared } \\
\text { (submitted to the } \\
\text { crioconcentration } \\
\text { method-ice 5) and } \\
\text { analyzed for the study. }\end{array}$ \\
\hline \multicolumn{12}{|c|}{ Sesame seed-based beverage } \\
\hline Reis, 2019 & Water (distilled), sesame seeds. & $139^{\mathrm{b}}$ & $12.77^{\mathrm{b}}$ & $5.55^{\mathrm{b}}$ & $7.26^{\mathrm{b}}$ & - & - & - & - & - & $\begin{array}{l}\text { Beverage prepared and } \\
\text { analyzed for the study. }\end{array}$ \\
\hline Reis, 2019 & $\begin{array}{l}\text { Water (distilled), sesame seeds, } \\
\text { maltodextrin }(10.00 \%) .\end{array}$ & $170^{\mathrm{b}}$ & $22.29^{b}$ & $5.23^{b}$ & $6.69^{b}$ & - & - & - & - & - & $\begin{array}{l}\text { Beverage prepared and } \\
\text { analyzed for the study. }\end{array}$ \\
\hline
\end{tabular}


Table 1. Cont.

\begin{tabular}{|c|c|c|c|c|c|c|c|c|c|c|c|}
\hline Authors and Year & Ingredients & $\begin{array}{l}\text { Energy } \\
\text { (Kcal) }\end{array}$ & $\begin{array}{c}\mathrm{CHO} \\
(\mathrm{g})\end{array}$ & $\begin{array}{l}\text { Protein } \\
\text { (g) }\end{array}$ & $\begin{array}{l}\text { Lipid } \\
\text { (g) }\end{array}$ & $\begin{array}{l}\text { Dietary } \\
\text { Fiber (g) }\end{array}$ & $\begin{array}{c}\mathrm{Ca} \\
(\mathrm{mg})\end{array}$ & $\begin{array}{c}\mathrm{Fe} \\
(\mathrm{mg})\end{array}$ & $\begin{array}{c}\mathrm{Mg} \\
(\mathrm{mg})\end{array}$ & $\begin{array}{c}\mathrm{Na} \\
(\mathrm{mg})\end{array}$ & $\begin{array}{l}\text { Specifications on the } \\
\text { Origin of the Beverage/ } \\
\text { Where the Nutritional } \\
\text { Data Were Obtained }\end{array}$ \\
\hline \multicolumn{12}{|c|}{ Soy-based beverage } \\
\hline Barros, 2012 & Water, soybean (cultivar Embrapa BRS-213). & $44^{\mathrm{b}}$ & $1.44^{\mathrm{b}}$ & $4.50^{\mathrm{b}}$ & $2.31^{\mathrm{b}}$ & - & - & - & - & - & $\begin{array}{l}\text { Beverage prepared } \\
\text { (control) and analyzed for } \\
\text { the study. }\end{array}$ \\
\hline Barros, 2012 & $\begin{array}{c}\text { Water, soybean (cultivar Embrapa } \\
\text { BRS-213), tocopherol. }\end{array}$ & $51^{\mathrm{b}}$ & $1.53^{\mathrm{b}}$ & $4.83^{\mathrm{b}}$ & $2.83^{b}$ & - & - & - & - & - & $\begin{array}{l}\text { Beverage prepared } \\
\text { (subjected to } 5.00 \mathrm{kGy} \\
\text { of irradiation } \\
\text { with tocopherol } \\
\text { supplementation) and } \\
\text { analyzed for the study. }\end{array}$ \\
\hline Barros, 2012 & $\begin{array}{c}\text { Water, soybean (cultivar Embrapa } \\
\text { BRS-213), tocopherol. }\end{array}$ & $54^{\mathrm{b}}$ & $1.70^{\mathrm{b}}$ & $4.99^{\mathrm{b}}$ & $2.99^{b}$ & - & - & - & - & - & $\begin{array}{l}\text { Beverage prepared } \\
\text { (subjected to a } \\
\text { temperature of } 80^{\circ} \mathrm{C} \\
\text { with tocopherol } \\
\text { supplementation) and } \\
\text { analyzed for the study. }\end{array}$ \\
\hline Barros, 2012 & $\begin{array}{c}\text { Water, soybean (cultivar Embrapa } \\
\text { BRS-258), tocopherol. }\end{array}$ & $51^{\mathrm{b}}$ & $1.69^{\mathrm{b}}$ & $4.74^{\mathrm{b}}$ & $2.79^{b}$ & - & - & - & - & - & $\begin{array}{l}\text { Beverage prepared } \\
\text { (subjected to } 5.00 \mathrm{kGy} \\
\text { of irradiation } \\
\text { with tocopherol } \\
\text { supplementation) and } \\
\text { analyzed for the study. }\end{array}$ \\
\hline Barros, 2012 & $\begin{array}{c}\text { Water, soybean (cultivar Embrapa } \\
\text { BRS-258), tocopherol. }\end{array}$ & $51^{b}$ & $1.67^{\mathrm{b}}$ & $4.57^{\mathrm{b}}$ & $2.94^{\mathrm{b}}$ & - & - & - & - & - & $\begin{array}{l}\text { Beverage prepared } \\
\text { (subjected to a } \\
\text { temperature of } 80^{\circ} \mathrm{C} \\
\text { with tocopherol } \\
\text { supplementation) and } \\
\text { analyzed for the study. }\end{array}$ \\
\hline Barros, 2012 & Water, soybean (cultivar Embrapa Emb-48). & $50^{b}$ & $1.87^{\mathrm{b}}$ & $4.28^{\mathrm{b}}$ & $2.88^{\mathrm{b}}$ & - & - & - & - & - & $\begin{array}{l}\text { Beverage prepared } \\
\text { (control) and analyzed for } \\
\text { the study }\end{array}$ \\
\hline
\end{tabular}


Table 1. Cont.

\begin{tabular}{|c|c|c|c|c|c|c|c|c|c|c|c|}
\hline Authors and Year & Ingredients & $\begin{array}{l}\text { Energy } \\
\text { (Kcal) }\end{array}$ & $\underset{(\mathrm{g})}{\mathrm{CHO}}$ & $\begin{array}{l}\text { Protein } \\
\text { (g) }\end{array}$ & $\begin{array}{l}\text { Lipid } \\
\text { (g) }\end{array}$ & $\begin{array}{l}\text { Dietary } \\
\text { Fiber }(\mathrm{g})\end{array}$ & $\begin{array}{c}\mathrm{Ca} \\
(\mathrm{mg})\end{array}$ & $\begin{array}{c}\mathrm{Fe} \\
(\mathrm{mg})\end{array}$ & $\underset{(\mathrm{mg})}{\mathrm{Mg}}$ & $\begin{array}{c}\mathrm{Na} \\
(\mathrm{mg})\end{array}$ & $\begin{array}{l}\text { Specifications on the } \\
\text { Origin of the Beverage/ } \\
\text { Where the Nutritional } \\
\text { Data Were Obtained }\end{array}$ \\
\hline Barros, 2012 & $\begin{array}{l}\text { Water, soybean (cultivar Embrapa } \\
\text { Emb-48), tocopherol. }\end{array}$ & $50^{\mathrm{b}}$ & $1.80^{\mathrm{b}}$ & $4.25^{b}$ & $2.82^{b}$ & - & - & - & - & - & $\begin{array}{l}\text { Beverage prepared } \\
\text { (subjected to } 5.00 \mathrm{kGy} \\
\text { of irradiation } \\
\text { with tocopherol } \\
\text { supplementation) and } \\
\text { analyzed for the study. }\end{array}$ \\
\hline Barros, 2016 & $\begin{array}{c}\text { Water, soybean (cultivar Embrapa BRS-213), } \\
\text { acacia/arabic gum (3.00\%), neutral alloy (guar and } \\
\text { carboxymethylcellulose) (1.00\%), vanilla essence } \\
(0.20 \%), \text { tocopherol, ascorbic acid, concentrated } \\
\text { apple juice. }\end{array}$ & $61^{\mathrm{b}}$ & $12.38^{\mathrm{b}}$ & $1.32^{\mathrm{b}}$ & $0.65^{\mathrm{b}}$ & - & - & - & - & - & $\begin{array}{l}\text { Beverage prepared } \\
\text { (control) and analyzed for } \\
\text { the study. }\end{array}$ \\
\hline Barros, 2016 & $\begin{array}{c}\text { Water, soybean (cultivar Embrapa BRS-213), } \\
\text { acacia/arabic gum }(3.00 \%), \text { neutral alloy (guar and } \\
\text { carboxymethylcellulose) }(1.00 \% \text {, vanilla essence } \\
(0.20 \%), \text { tocopherol, ascorbic acid, concentrated } \\
\text { apple juice. }\end{array}$ & $61^{\mathrm{b}}$ & $12.18^{b}$ & $1.12^{\mathrm{b}}$ & $0.82^{b}$ & - & - & - & - & - & $\begin{array}{l}\text { Beverage prepared } \\
\text { (subjected to } 4.00 \mathrm{kGy} \text { of } \\
\text { gamma radiation) and } \\
\text { analyzed for the study. }\end{array}$ \\
\hline Barros, 2016 & $\begin{array}{c}\text { Water, soybean (cultivar Embrapa BRS-213), } \\
\text { acacia/arabic gum (3.00\%), neutral alloy (guar and } \\
\text { carboxymethylcellulose) }(1.00 \%) \text {, vanilla essence } \\
(0.20 \%) \text {, tocopherol, ascorbic acid, concentrated } \\
\text { apple juice. }\end{array}$ & $60^{\mathrm{b}}$ & $12.41^{\mathrm{b}}$ & $0.99^{b}$ & $0.70^{\mathrm{b}}$ & - & - & - & - & - & $\begin{array}{l}\text { Beverage prepared } \\
\text { (subjected to } 8.00 \mathrm{kGy} \text { of } \\
\text { gamma radiation) and } \\
\text { analyzed for the study. }\end{array}$ \\
\hline
\end{tabular}


Table 1. Cont.

\begin{tabular}{|c|c|c|c|c|c|c|c|c|c|c|c|}
\hline Authors and Year & Ingredients & $\begin{array}{l}\text { Energy } \\
\text { (Kcal) }\end{array}$ & $\begin{array}{c}\mathrm{CHO} \\
(\mathrm{g})\end{array}$ & $\begin{array}{l}\text { Protein } \\
\text { (g) }\end{array}$ & $\begin{array}{l}\text { Lipid } \\
\text { (g) }\end{array}$ & $\begin{array}{l}\text { Dietary } \\
\text { Fiber (g) }\end{array}$ & $\begin{array}{l}\mathrm{Ca} \\
(\mathrm{mg})\end{array}$ & $\begin{array}{c}\mathrm{Fe} \\
(\mathrm{mg})\end{array}$ & $\underset{(\mathrm{mg})}{\mathrm{Mg}}$ & $\begin{array}{c}\mathrm{Na} \\
(\mathrm{mg})\end{array}$ & $\begin{array}{l}\text { Specifications on the } \\
\text { Origin of the Beverage/ } \\
\text { Where the Nutritional } \\
\text { Data Were Obtained }\end{array}$ \\
\hline $\begin{array}{l}\text { Barros and Venturini } \\
\text { Filho, } 2016\end{array}$ & Water, soybean. & $26^{\mathrm{b}}$ & $0.41^{\mathrm{b}}$ & $2.77^{b}$ & $1.44^{\mathrm{b}}$ & - & - & - & - & - & $\begin{array}{l}\text { Beverage prepared } \\
\text { (cold grinding } \\
\text { method-aluminum } \\
\text { cauldron) and analyzed } \\
\text { for the study. }\end{array}$ \\
\hline $\begin{array}{l}\text { Barros and Venturini } \\
\text { Filho, } 2016\end{array}$ & Water, soybean. & $31^{b}$ & $1.54^{\mathrm{b}}$ & $3.18^{\mathrm{b}}$ & $1.44^{\mathrm{b}}$ & - & - & - & - & - & $\begin{array}{c}\text { Beverage prepared } \\
\text { (hot grinding } \\
\text { method-mechanical cow) } \\
\text { and analyzed for } \\
\text { the study. }\end{array}$ \\
\hline $\begin{array}{l}\text { Barros and Venturini } \\
\text { Filho, } 2016\end{array}$ & Water, soybean, sugar. & $62^{\mathrm{b}}$ & $10.27^{b}$ & $2.57^{\mathrm{b}}$ & $1.23^{b}$ & - & - & - & - & - & $\begin{array}{l}\text { Beverage prepared } \\
\text { (cold grinding } \\
\text { method-aluminum } \\
\text { cauldron) and analyzed } \\
\text { for the study. }\end{array}$ \\
\hline $\begin{array}{l}\text { Barros and Venturini } \\
\text { Filho, } 2016\end{array}$ & Water, soybean, sugar. & $67^{b}$ & $10.37^{\mathrm{b}}$ & $3.08^{b}$ & $1.34^{\mathrm{b}}$ & - & - & - & - & - & $\begin{array}{l}\text { Beverage prepared } \\
\text { (hot grinding } \\
\text { method-mechanical cow) } \\
\text { and analyzed for } \\
\text { the study. }\end{array}$ \\
\hline $\begin{array}{l}\text { Chalupa-Krebzdak } \\
\text { et al., } 2018\end{array}$ & $\begin{array}{l}\text { Soymilk } 97.20 \% \text { (purified water, soy beans), coconut } \\
\text { oil, sugar, water, salt, glycerin mono fatty acid ester, } \\
\text { sodium bicarbonate. }\end{array}$ & 58 & 2.63 & 3.16 & 3.68 & - & 0.00 & - & - & - & $\begin{array}{l}\text { USDA Food Composition } \\
\text { Database. }\end{array}$ \\
\hline $\begin{array}{l}\text { Chalupa-Krebzdak } \\
\text { et al., } 2018\end{array}$ & $\begin{array}{l}\text { Organic soymilk (filtered water, whole organic soy } \\
\text { beans), calcium carbonate, organic locust bean gum, } \\
\text { sea salt, natural flavours, gellan gum, vitamin A } \\
\text { palmitate, ergocalciferol (vitamin D2), riboflavin } \\
\text { (vitamin B2), cyanoconalamin (vitamin B12). }\end{array}$ & 33 & 1.67 & 2.92 & 1.67 & - & 125.00 & - & - & - & $\begin{array}{l}\text { USDA Food Composition } \\
\text { Database. }\end{array}$ \\
\hline $\begin{array}{l}\text { Chalupa-Krebzdak } \\
\text { et al., } 2018\end{array}$ & $\begin{array}{c}\text { Soy milk (filtered water, soy beans), cane sugar, } \\
\text { contains } 2.00 \% \text { or less of: vitamin and mineral blend } \\
\text { (calcium carbonate, vitamin A palmitate, vitamin } \\
\text { D2, riboflavin B2, vitamin B12), sea salt, natural } \\
\text { flavor, gellan gum. }\end{array}$ & 42 & 5.00 & 2.50 & 1.46 & - & 188.00 & - & - & - & $\begin{array}{l}\text { USDA Food Composition } \\
\text { Database. }\end{array}$ \\
\hline
\end{tabular}


Table 1. Cont.

\begin{tabular}{|c|c|c|c|c|c|c|c|c|c|c|c|}
\hline Authors and Year & Ingredients & $\begin{array}{c}\text { Energy } \\
\text { (Kcal) }\end{array}$ & $\underset{(\mathrm{g})}{\mathrm{CHO}}$ & $\begin{array}{l}\text { Protein } \\
\text { (g) }\end{array}$ & $\begin{array}{l}\text { Lipid } \\
\text { (g) }\end{array}$ & $\begin{array}{l}\text { Dietary } \\
\text { Fiber (g) }\end{array}$ & $\begin{array}{c}\mathrm{Ca} \\
(\mathrm{mg})\end{array}$ & $\begin{array}{c}\mathrm{Fe} \\
(\mathrm{mg})\end{array}$ & $\underset{(m g)}{M g}$ & $\begin{array}{c}\mathrm{Na} \\
(\mathrm{mg})\end{array}$ & $\begin{array}{l}\text { Specifications on the } \\
\text { Origin of the Beverage/ } \\
\text { Where the Nutritional } \\
\text { Data Were Obtained }\end{array}$ \\
\hline $\begin{array}{l}\text { Chalupa-Krebzdak } \\
\text { et al., } 2018\end{array}$ & $\begin{array}{l}\text { Filtered water, organic whole soybeans, organic fair } \\
\text { trade unrefined cane sugar, calcium carbonate, salt, } \\
\text { organic fair trade vanilla flavor, vitamin A palmitate, } \\
\text { gellan gum, riboflavin (vitamin B2), vitamin B12. }\end{array}$ & 46 & 4.58 & 2.92 & 1.67 & - & 125.00 & - & - & - & $\begin{array}{l}\text { USDA Food Composition } \\
\text { Database. }\end{array}$ \\
\hline $\begin{array}{l}\text { Chalupa-Krebzdak } \\
\text { et al., } 2018\end{array}$ & $\begin{array}{c}\text { Filtered water, whole organic soybeans, evaporated } \\
\text { organic cane juice, calcium carbonate, organic } \\
\text { natural flavours, sea salt, xanthan gum, carrageenan, } \\
\text { vitamin A palmitate, riboflavin (B2), vitamin D2, } \\
\text { vitamin B12. }\end{array}$ & 42 & 3.75 & 2.92 & 1.67 & - & 125.00 & - & - & - & $\begin{array}{l}\text { USDA Food Composition } \\
\text { Database. }\end{array}$ \\
\hline Decloedt et al., 2018 & $\begin{array}{l}\text { Water, soybeans (pealed), tricalcium phosphate } \\
(5.90 \%) \text {, monopotassium phosphate (acid regulator), } \\
\text { salt, aromas, sugar, gellan gum. }\end{array}$ & 39 & 2.50 & 3.00 & 1.80 & 0.50 & 120.00 & - & - & $44.00^{\mathrm{a}}$ & $\begin{array}{l}\text { Nutritional values } \\
\text { obtained from the label of } \\
\text { the drinks that } \\
\text { were purchased. }\end{array}$ \\
\hline Ferreira, 2011 & Water, soybeans (1:8 soy:water ratio). & $29^{b}$ & $3.21^{\mathrm{b}}$ & $3.39^{\mathrm{b}}$ & $1.13^{\mathrm{b}}$ & - & - & - & - & - & $\begin{array}{l}\text { Beverage prepared and } \\
\text { analyzed for the study. }\end{array}$ \\
\hline Ferreira, 2011 & Water, soybeans (1:10 soy: water ratio). & $28^{\mathrm{b}}$ & $1.41^{\mathrm{b}}$ & $1.62^{\mathrm{b}}$ & $0.98^{\mathrm{b}}$ & - & - & - & - & - & $\begin{array}{l}\text { Beverage prepared and } \\
\text { analyzed for the study. }\end{array}$ \\
\hline Ferreira, 2011 & Water, soybeans (1:12 soy: water ratio). & $16^{\mathrm{b}}$ & $1.18^{\mathrm{b}}$ & $1.55^{\mathrm{b}}$ & $0.51^{\mathrm{b}}$ & - & - & - & - & - & $\begin{array}{l}\text { Beverage prepared and } \\
\text { analyzed for the study. }\end{array}$ \\
\hline Hajirostamloo, 2009 & Water, soybean. & $33^{b}$ & $1.86^{\mathrm{b}}$ & $2.82^{b}$ & $1.96^{\mathrm{b}}$ & $1.33^{\mathrm{b}}$ & $4.11^{\mathrm{b}}$ & $0.59^{b}$ & - & - & $\begin{array}{l}\text { Beverage prepared and } \\
\text { analyzed for the stud.y }\end{array}$ \\
\hline $\begin{array}{l}\text { Martínez-Padilla et al., } \\
2020\end{array}$ & Water, shelled soybean $(7.20 \%)$. & 35 & 0.10 & 3.70 & 2.10 & - & - & - & - & - & $\begin{array}{l}\text { Nutritional values } \\
\text { obtained from the label. } \\
\text { Brand name: Naturli. }\end{array}$ \\
\hline Nti et al., 2016 & Water, soybeans, salt $(0.20 \%)$. & $50^{b}$ & $6.85^{b}$ & $2.60^{\mathrm{b}}$ & $1.62^{b}$ & - & $19.00^{\mathrm{b}}$ & $0.51^{b}$ & $22.59^{b}$ & $2.57^{\mathrm{b}}$ & $\begin{array}{l}\text { Beverage prepared and } \\
\text { analyzed for the study. }\end{array}$ \\
\hline $\begin{array}{l}\text { Scholz-Ahrens et al., } \\
2020\end{array}$ & $\begin{array}{l}\text { Water, dehulled soyabeans }(7.20 \%) \text {, apple } \\
\text { concentrate }(3.30 \%) \text {, algae Lithothamnium } \\
\text { calcareum }(0.40 \%) \text {, sea salt. }\end{array}$ & $46^{\mathrm{b}}$ & $2.46^{\mathrm{b}}$ & $3.80^{\mathrm{b}}$ & $2.16^{\mathrm{b}}$ & - & $\underset{\mathrm{b}}{123.24}$ & - & - & - & $\begin{array}{l}\text { Nutritional values } \\
\text { obtained from the label. } \\
\text { Brand name: Provamel. }\end{array}$ \\
\hline $\begin{array}{l}\text { Scholz-Ahrens et al., } \\
2020\end{array}$ & $\begin{array}{l}\text { Water, raw cane sugar, dehulled soyabeans }(5.80 \%) \text {, } \\
\text { fat reduced cocoa }(1.30 \%) \text {, chocolate }(1.10 \%) \text {, sea salt. }\end{array}$ & $69^{b}$ & $8.32^{b}$ & $3.49^{\mathrm{b}}$ & $2.26^{\mathrm{b}}$ & - & ND & - & - & - & $\begin{array}{l}\text { Nutritional values } \\
\text { obtained from the label. } \\
\text { Brand name: Provamel. }\end{array}$ \\
\hline $\begin{array}{l}\text { Uliana and Venturini } \\
\text { Filho, } 2010\end{array}$ & Water, soybean. & $33^{b}$ & $2.11^{b}$ & $2.82^{b}$ & $1.43^{b}$ & - & - & - & - & - & $\begin{array}{l}\text { Beverage prepared and } \\
\text { analyzed for the study. }\end{array}$ \\
\hline
\end{tabular}


Table 1. Cont.

\begin{tabular}{|c|c|c|c|c|c|c|c|c|c|c|c|}
\hline Authors and Year & Ingredients & $\begin{array}{l}\text { Energy } \\
\text { (Kcal) }\end{array}$ & $\underset{(\mathrm{g})}{\mathrm{CHO}}$ & $\begin{array}{l}\text { Protein } \\
\quad(g)\end{array}$ & $\begin{array}{l}\text { Lipid } \\
\text { (g) }\end{array}$ & $\begin{array}{l}\text { Dietary } \\
\text { Fiber (g) }\end{array}$ & $\begin{array}{c}\mathrm{Ca} \\
(\mathrm{mg})\end{array}$ & $\begin{array}{c}\mathrm{Fe} \\
(\mathrm{mg})\end{array}$ & $\begin{array}{c}\mathrm{Mg} \\
(\mathrm{mg})\end{array}$ & $\begin{array}{c}\mathrm{Na} \\
(\mathrm{mg})\end{array}$ & $\begin{array}{l}\text { Specifications on the } \\
\text { Origin of the Beverage/ } \\
\text { Where the Nutritional } \\
\text { Data Were Obtained }\end{array}$ \\
\hline $\begin{array}{l}\text { Uliana and Venturini } \\
\text { Filho, } 2010\end{array}$ & Water, soybean. & $31^{\mathrm{b}}$ & $1.80^{\mathrm{b}}$ & $2.88^{\mathrm{b}}$ & $1.39^{\mathrm{b}}$ & - & - & - & - & - & $\begin{array}{l}\text { Beverage prepared and } \\
\text { analyzed for the study. }\end{array}$ \\
\hline $\begin{array}{l}\text { Uliana and Venturini } \\
\text { Filho, } 2010\end{array}$ & Water, soybean. & $31^{\mathrm{b}}$ & $1.85^{\mathrm{b}}$ & $2.82^{\mathrm{b}}$ & $1.34^{\mathrm{b}}$ & - & - & - & - & - & $\begin{array}{l}\text { Beverage prepared and } \\
\text { analyzed for the study. }\end{array}$ \\
\hline \multicolumn{12}{|c|}{ Spelt-based beverage } \\
\hline \multicolumn{12}{|c|}{ Sunflower seed-based beverage } \\
\hline Blum et al., 2016 & Water (distilled), germinated sunflower seeds. & $21^{\mathrm{b}}$ & $0.00^{\mathrm{b}}$ & $0.77^{\mathrm{b}}$ & $2.00^{\mathrm{b}}$ & $1.20^{\mathrm{b}}$ & $0.16^{\mathrm{b}}$ & - & - & $1.88^{\mathrm{b}}$ & $\begin{array}{l}\text { Beverage prepared and } \\
\text { analyzed for the study. }\end{array}$ \\
\hline \multicolumn{12}{|c|}{ Tucumã almond-based beverage } \\
\hline Silva et al., 2015 & Water, tucumã almond flour. & $6^{\mathrm{b}}$ & $0.44^{\mathrm{b}}$ & $0.06^{\mathrm{b}}$ & $0.45^{\mathrm{b}}$ & - & - & - & - & - & $\begin{array}{l}\text { Beverage prepared } \\
(\text { extraction temperature of } \\
55^{\circ} \mathrm{C} \text { ) and analyzed for } \\
\text { the study. }\end{array}$ \\
\hline Silva et al., 2015 & Water, tucumã almond flour. & $7^{b}$ & $0.47^{\mathrm{b}}$ & $0.07^{\mathrm{b}}$ & $0.51^{\mathrm{b}}$ & - & - & - & - & - & $\begin{array}{c}\text { Beverage prepared } \\
(\text { extraction temperature of } \\
75^{\circ} \mathrm{C} \text { ) and analyzed for } \\
\text { the study. }\end{array}$ \\
\hline Silva et al., 2015 & Water, tucumã almond flour. & $10^{\mathrm{b}}$ & $0.38^{\mathrm{b}}$ & $0.07^{b}$ & $0.92^{b}$ & - & - & - & - & - & $\begin{array}{c}\text { Beverage prepared } \\
(\text { extraction temperature of } \\
100^{\circ} \mathrm{C} \text { ) and analyzed for } \\
\text { the study. }\end{array}$ \\
\hline \multicolumn{12}{|c|}{ Yam-based beverage } \\
\hline Araújo, 2015 & Water, yam. & $10^{\mathrm{b}}$ & $2.40^{\mathrm{b}}$ & $0.17^{b}$ & $0.01^{\mathrm{b}}$ & $0.18^{\mathrm{b}}$ & $3.59^{\mathrm{b}}$ & $0.06^{\mathrm{b}}$ & - & $0.82^{b}$ & $\begin{array}{l}\text { Beverage prepared for the } \\
\text { study. Nutritional values } \\
\text { taken from the TACO and } \\
\text { the IBGE food } \\
\text { composition table. }\end{array}$ \\
\hline
\end{tabular}

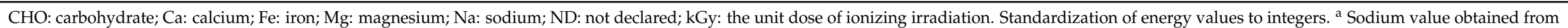
the salt value ( $1 \mathrm{~g}$ of salt is equivalent to $400 \mathrm{mg}$ of sodium). ${ }^{\mathrm{b}}$ Values converted to standard serving size (100 $\left.\mathrm{mL}\right)$. 


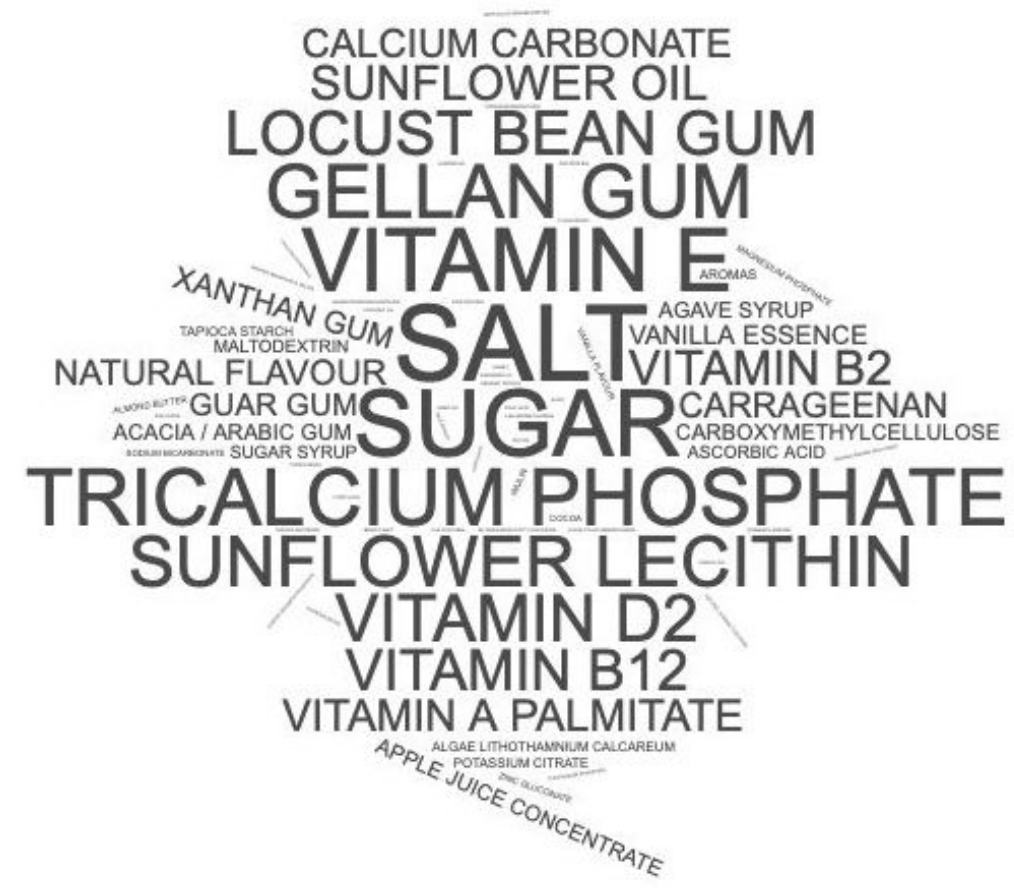

Figure 1. Word cloud of added ingredients from plant-based beverages from scientific studies selected according to the inclusion and exclusion criteria. Words are presented according to their proportional frequencies among all samples, given that greater sizes correspond to greater frequencies.

\section{Discussion}

\subsection{Nutritional Content Variations}

This review confirmed that plant-based beverages' nutritional content depends on different aspects, such as the type of raw material used to produce it, the process, and the added ingredients [11,21].

\subsubsection{Processing Performed}

The sapucaia nut-based beverage (the sapucaia nut (Lecythis pisonis) is a Brazilian species found in the Amazon and Atlantic Forest) was subjected to crioconcentration in block (method of total freezing followed by partial thawing of the solution, under gravitational separation), capable of concentrating solid matter by removing water in ice form [47]. This process concentrates nutritional compounds, such as protein, carbohydrate, calcium, and magnesium, influencing the beverage's nutritional content [47]. This concentrated water-soluble extract can be used as an ingredient for other food products to improve their nutritional content as selenium, in which the concentrated extract could reach $10,037 \mu \mathrm{g} / \mathrm{g}$ [47].

The hot grinding method-capable of inactivating the lipoxygenase enzyme of the water-soluble soy extract that causes its typical "beany" flavor-[63] and the cold grinding method (traditional oriental method) were performed to obtain water-soluble soy extract [44]. The cold-extracted beverages had a higher moisture content and, consequently, lower concentrations of proteins and total solids than the hot-extracted beverages [44].

A study analyzed the effect of physical and antioxidant treatments on the lipoxygenase enzyme activity in a soy-based beverage [43]. The author observed that the protein and lipid content of BRS-213 cultivar's beverages subjected to physical treatments (irradiation-5.00 kGy; thermal- $80^{\circ} \mathrm{C}$ ) with tocopherol supplementation were higher than the control sample. The study showed that adequate heat treatment increases lipids and proteins content, as well as their solubility and digestibility [43].

The physical treatments (irradiation-5.00 kGy; thermal-80 ${ }^{\circ} \mathrm{C}$ ) with tocopherol supplementation in BRS-213 cultivar's beverages also increased the macronutrient concentration compared to the control sample due to lower moisture content [43]. Furthermore, 
the beverages produced with the EMB-48 soy cultivar subjected to heat treatment had a significant increase in lipid and protein content [43]. The hydrothermal processing of the soy-based beverage caused a rupture in the soy cell wall, allowing the passage of lipids and proteins, which was responsible for increasing the content of these nutrients [43].

As for irradiation (conservation technique) to which soy-based beverages were subjected, a study [42] verified a significant reduction in the protein content when increasing the dose of gamma radiation. Deamination (release of the amine group from the rupture of the peptide bonds of the amino acid, forming ammonia) was mentioned as a possible cause [42]. However, this process may have improved the protein quality since deamination makes it more hydrophilic, increasing its solubility and digestibility [42].

Another aspect in producing plant-based beverages capable of influencing its nutritional content is the raw material:water ratio. A study [48] found higher macronutrient and energy concentration in the soy-based beverage with a 1:8 ratio (soy:water) than in those with a 1:10 and 1:12 ratio. These results were expected since water dilution implies nutrients dilution in the final product.

\subsubsection{Added Ingredients}

In the production of plant-based beverages, the addition of other ingredients may improve the nutritional profile, the functional or sensory properties of the beverage [22,64]. Salt, sugar, syrups, flavorings, vanilla essence, cocoa, apple juice concentrated, and others are incorporated to improve sensory aspects, mainly the flavor [42,64]. In addition to enhancing flavor, salt and sugar are usually added in beverages and foods to improve texture or shelf-life [65].

Cow's milk presents a mild and typical flavor, neither sweet nor salty, characterized mainly by the balance between lactose and salt [4]. Therefore, it is common to find salt in plant-based beverages, as well as ingredients for a sweet flavor (sugar, maltodextrin, apple juice, agave syrup, vanilla extract). It is important to highlight that the use of ingredients like sucrose, maltodextrin, and fructose might negatively impact the glycemic index and salt also might impair the nutritional quality. The consumption of foods with low $(<55)$ and medium (56-69) glycemic index is recommended, especially to control blood glucose levels $[20,23]$. A low glycemic load diet is related to a lower risk of diabetes, cardiovascular disease, and obesity [66].

Sugar is often used to improve sensory quality but influences the nutritional quality negatively. In the studies by Barros and Venturini Filho [44] and Karimidastjerd and Kilic-Akyilmaz [60], the plant-based beverages with added sugar are more caloric and with higher amounts of carbohydrate than those without added sugar. In the sesamebased beverages studied by Reis [34], the carbohydrate amount of the sample added with maltodextrin is almost twice the amount of the pure sesame-based beverage. Consequently, the sample with maltodextrin is more caloric [34].

Other ingredients commonly found in plant-based beverages are vegetable oils, such as sunflower oil. Martínez-Padilla et al. [53] pointed out that the addition of these oils can provide a smooth mouthfeel similar to that of cow's milk, and Aydar et al. [64] mentioned its use in order to improve the silky aspect.

A study [22] showed that it is possible to add starch in plant-based beverages as a plant-derived thickening agent and pectin or locust bean gum to improve the texture. Reis [34] used maltodextrin to improve the stability of the beverage and Sethi et al. [19] referred to the use of sodium bicarbonate as an alkalizing agent, which might prevent destabilization by sedimentation of solid particles in the beverage.

A study [39] used enzymes in the quinoa-based beverage preparation, with the addition of the Termamyl enzyme for the dextrinization process and the amyloglucosidase enzyme for saccharification. In food industry, inulin can be used as a texture modifier, sugar or fat substitute, and it also has a prebiotic effect on the human organism [67].

Due to the nutritional composition of most plant-based beverages (not similar to that of cow's milk-high in protein and calcium) and nutritional losses during the processing 
of the raw material, in plant-based beverages the addition of vitamins (e.g., A, D, E, B2, folic acid, B12), minerals (e.g., calcium, zinc) and proteins (usually isolated or extracted from sources, for example, peas or soy) is frequent, trying to achieve a similar composition to cow's milk [68]. However, other vitamins and minerals are added with different functions. Barros [43] added tocopherol (vitamin E) with the function of antioxidant treatment of beverages.

Zinc gluconate, zinc oxide, calcium carbonate, tricalcium phosphate and monocalcium phosphate are some of the micronutrient compounds used as food fortifiers [69]. Algae Lithothamnium calcareum is mentioned by Scholz-Ahrens et al. [54] as another source of calcium added to plant-based beverages. Although these beverages are often fortified, the added nutrients are not always bioavailable as those naturally present in milk [68].

Tricalcium phosphate can be used for several functions as an acidity regulator, buffer, anticaking agent, clouding agent, emulsifying, firming agent, flour treatment agent, humectant, moisture-retention agent, raising agent, stabilizer and texturizing agent; calcium carbonate can be used as an acidity regulator, anticaking agent, surface colorant, firming agent, dough conditioner, and stabilizer [70]. Some of these functions are important in plant-based beverages, but they were not discussed in the studies that use them.

The function of gums as food additives can also be used in plant-based beverages. In this sense, gellan gum is used as a gelling agent, stabilizer, and thickener; locust (or carob) bean gum and guar gum are used as an emulsifier, stabilizer, and thickener; xanthan gum as an emulsifier, foaming agent, stabilizer and thickener; gum arabic (acacia gum) is used as a bulking agent, carrier, emulsifier, glazing agent, stabilizer and thickener [70].

The technological purposes of other food additives that appeared in the beverages ingredients lists are carrageenan (bulking agent, carrier, emulsifier, gelling agent, glazing agent, coating agent, humectant, stabilizer and thickener); lecithin (antioxidant and emulsifier); ascorbic acid (acidity regulator, antioxidant, flour treatment agent and sequestrant); citric acid (acidity regulator, antioxidant, color retention agent, and sequestrant); sodium carboxymethyl cellulose (bulking agent, emulsifier, suspension agent, firming agent, gelling agent, glazing agent, coating agent, humectant, stabilizer and thickener); sodium potassium hexametaphosphate (acidity regulator; emulsifier, moisture-retention agent, raising agent, sequestrant, stabilizer and texturizing agent); sodium metabisulfite (antioxidant, bleaching agent, flour treatment agent and preservative); potassium citrate (acidity regulator, emulsifying salt, sequestrant and stabilizer); potassium phosphate (acidity regulator, buffer, emulsifier, emulsifying salt, humectant, moisture-retention agent, sequestrant, stabilizer and texturizing agent); magnesium phosphate (acidity regulator, anticaking agent, emulsifying salt, raising agent, stabilizer and thickener); sucrose esters of fatty acids (emulsifier, foaming agent, glazing agent and stabilizer); mono- and di-glycerides of fatty acids (antifoaming agent, emulsifier, glazing agent, surface-finishing agent and stabilizer) [70].

\subsection{Comparison of Nutritional Composition: Plant-Based Beverages and Cow's Milk}

As a reference for comparing the nutritional composition of the types of plant-based beverages, the nutritional composition (per $100 \mathrm{~g}$ ) of a whole cow's milk obtained from USDA are energy (64 Kcal), carbohydrate $(4.65 \mathrm{~g})$, protein $(3.28 \mathrm{~g})$, lipid $(3.66 \mathrm{~g})$, calcium (119.00 mg), iron (0.05 mg), magnesium (13.00 mg) and sodium (49.00 mg) [71]. Due to the FAO [71] document not showing the dietary fiber value of this whole cow's milk, this nutrient's reference was established as $0.00 \mathrm{~g} / 100 \mathrm{~mL}$ according to other milk present in the USDA FoodData Central [72].

\subsubsection{Energy}

The energy value comes from macronutrients (carbohydrate, protein, and lipid) [23]. Considering the energy value of the cow's milk mentioned above converted to the standard serving size of this study $(66 \mathrm{Kcal} / 100 \mathrm{~mL})$, most plant-based beverages $(86.07 \%, n=105)$ are less caloric than the cow's milk using the same portion. Only one almond-based bever- 
age [36], the baru almond-based beverage [40], two cashew nut-based beverages [23,33], three coconut-based beverages [23,54], the groundnut-based beverage [49], one rice-based beverage [57], the safflower-based beverage [49], two sapucaia nut-based beverages [47], both sesame seed-based beverages [34], two soy-based beverages [44,54], and the speltbased beverage [54], presented a total energetic value ranging from 66 to $183 \mathrm{Kcal} / 100 \mathrm{~mL}$ equal or higher than the cow's milk.

\subsubsection{Carbohydrate}

Almost $27.87 \%(n=34)$ of the studied beverages were higher in carbohydrate content than the cow's milk (4.78 g/100 mL). Regarding differences in composition, the carbohydrate in cow's milk is mostly lactose-contributes to the use of vitamin D and the absorption of calcium, phosphorus and magnesium in the intestine-while plant-based beverages are lactose-free $[4,21,71]$ and flavor lactose substitutes most used are sugar, maltodextrin, apple juice, and agave syrup.

Regarding glycemic index (GI), Jeske et al. [66] evaluated the GI of bovine milk and commercial milk substitutes produced with almonds (Prunus dulcis), cashews (Anacardium occidentale), coconut (Cocos nucifera), hazelnut (Corylus avellana), hemp (Cannabis sativa), macadamia (Macadamia spp.), oat (Avena sativa), quinoa (Chenopodium quinoa), rice (Oryza spp.), and soy (Glycine max). The plant-based beverages GI ranged from 47.53 (Organic soya drink from Provamel) to 99.96 (Organic brown rice drink from Rude Health), while cow's milk (Clona Dairy Product Ltd., Clonakilty, Ireland) had GI equal to 46.93 [66]. Among the beverages analyzed by Jeske et al. [66], eight based on almond (Alpro), carob/almond, cashew, macadamia, quinoa and soy (Provamel, Sojade and Alpro-whole-bean) presented low GI as well as bovine milk, and six other based on almonds, hazelnut, hemp, oat, and soy had medium GI. The analyzed beverages based on coconut and rice, which contained mostly glucose, had a high GI [66].

The GI value is usually influenced by the type of sugar, with each type having a GI value [66]. Jeske et al. [66] presented the GI of maltose (105), sucrose (61), fructose (19), and lactose (46). Some of the plant-based beverage samples mentioned above had added sugar or sweeteners, such as agave syrup (present in cashew, macadamia and quinoa samples), apple concentrate (present in the soy sample from Provamel), sucrose (present in hazelnut, almond and soy original from Alpro) and maple syrup (present in the carob/almond sample) [66]. Agave syrup and apple concentrate are high in fructose, while sucrose and maple syrup contributed to high sucrose values [66]. Additionally, products that have ingredients rich in starch are high in glucose and/or maltose. Rice-based samples presented high maltose and glucose content and sample based on oat, which contains $\beta$-glucan capable of reducing GI, was high in maltose [66].

\subsubsection{Protein}

Of the plant-based beverages studied, $16.39 \%(n=20)$ have a higher amount of protein than the cow's milk (3.37 g/100 mL). Regarding protein quality, the Digestible Indispensable Amino Acid Score (DIAAS) is the recommended measure for analyzing it [73]. From the DIAAS cut-off values, the food can be characterized as "excellent/high" (value equal to or greater than 100) and "good/source" (between 75 and 99) [73].

The DIAAS method is indicated to replace the Protein Digestibility Corrected Amino Acid Score (PDCAAS) based on some considerations, such as using a single value of fecal crude protein digestibility in the PDCAAS method for correction for digestibility, while the DIAAS use "true ileal amino acid digestibility for the dietary indispensable amino acids" [73]. It is also pointed out an overvaluation of the protein quality of foods that contain antinutritional factors and an inadequate estimate of the protein quality of high-quality proteins due to a non-attribution of extra nutritional value by the PDCAAS method [73].

Chalupa-Krebzdak et al. [23], Scholz-Ahrens et al. [54], and Sousa and Kopf-Bolanz [65] showed DIAAS values for milk proteins and some plant-proteins (soy (Glycine max), rice 
(Oryza spp.), oats (Avena satina) and almond (Prunus dulcis)). In these studies, cow's milk has a higher DIAAS value than the plant-based proteins. Among the plant-proteins, soy presented DIAAS values (84.00-90.60) closest to cow's milk $[23,54,65]$. The DIAAS values of the other proteins presented were oats (54.00) [54,65], almond (40.00) [54] and rice (37.00-59.00) [23,54,65], not considered good nor excellent [73] as soy and cow's milk.

Proteins provide amino acids that perform essential functions in the body, such as structural, defense, transport, and regulatory [23,74]. The deficiency of essential amino acids, which must be ingested in the diet, can reduce protein synthesis and physiological and biochemical changes [74]. Thus, attention is needed when replacing milk with a plant-based beverage regarding protein, and a diet needs to be planned to guarantee an adequate aminogram.

\subsubsection{Lipid}

The percentage of plant-based beverages studied in which lipid content is higher than cow's milk (3.76 g/100 mL) is $13.11 \%(n=16)$. The lipid in cow's milk is mainly composed of saturated fatty acids, while plant-based beverages tend to have a higher content of unsaturated fatty acids and are cholesterol-free $[4,6,20,23,53]$. However, there may be exceptions, as mentioned by Chalupa-Krebzdak et al. [23], Craig and Fresán [20] and Vanga and Raghavan [6] the case of coconut-based beverage that has a high content of saturated fatty acids.

The high consumption of foods rich in saturated fatty acids and cholesterol is associated with an increased risk of developing cardiovascular diseases, while the consumption of polyunsaturated fatty acids corresponds to a risk reduction factor for these diseases [74]. Despite that, studies have shown that the consumption of dairy products does not negatively affect human cardiovascular health $[23,53,75]$. Chalupa-Krebzdak et al. [23] mentioned as possible cause that "many nutrients in dairy products that may offset the effects of potentially harmful levels of dietary saturated fatty acids".

The isocaloric replacement of saturated fatty acids for polyunsaturated has collaborated to reduce LDL and total cholesterol in humans [23,74]. In this sense, the lipid composition of the plant-based beverages does not have to be the same as that of cow's milk regarding the nutritional aspect [23] but is necessary to evaluate its need considering sensory and technological aspects. Chalupa-Krebzdak et al. [23] pointed out that replacing milk with plant-based beverages, except for some coconut-based beverages, can decrease the intake of saturated fatty acids in the diet.

Although coconut-based beverage has a high content of saturated fatty acids $[6,20,23]$, it contains medium-chain fatty acids (MCFAs) that can be converted into ketone compounds, which are favorable in brain functioning [14]. In addition, Vanga and Raghavan [6] report that lauric acid, which mainly contributes to raise high-density lipoprotein (HDL) cholesterol levels and, consequently, reduce levels in the bloodstream of low-density lipoprotein (LDL) cholesterol, is present in coconut fats.

\subsubsection{Dietary Fiber}

The consumption of dietary fiber might contribute to intestinal regulation, the reduction of blood cholesterol and glucose, and it is also associated with a lower incidence of diabetes, cardiovascular disease, gastrointestinal disorders and colon cancer [74,76]. Among the plant-based beverages that present dietary fiber data, most $(81.82 \%, n=18)$ have a higher dietary fiber content than cow's milk reference $(0.00 \mathrm{~g} / 100 \mathrm{~mL})$. In this sense, plant-based beverages have an advantage. Unfortunately, due to the studies' lack of data, it was not possible to link the fiber content and GI in the samples analyzed.

\subsubsection{Micronutrients}

Calcium is a nutrient that stands out in milk and is essential for bone and dental structure, muscle function, and nerve conduction [54,74]. The calcium values of plant-based beverages ranged from 0.00 to $1252.94 \mathrm{mg} / 100 \mathrm{~mL}$ and $44.44 \%(n=24)$ of the plant-based 
beverages were greater than the cow's milk $(122.21 \mathrm{mg} / 100 \mathrm{~mL})$. These beverages with a higher calcium content (except for the beverages based on sapucaia nut, which went through crioconcentration as previously mentioned), were all fortified with calcium.

As mentioned earlier about fortification, many commercial plant-based beverages are fortified with calcium to achieve cow's milk's amount [21]. Despite this, there are plant-based beverages that are not fortified. The substitution of milk for these non-fortified beverages when the diet is not balanced, calcium and other nutrients may be deficient [21].

Moreover, the bioavailability of the fortifier, and not only the amount of the nutrient, should also be taken into account [54]. As an example of calcium, Craig and Fresán [20] mention that calcium absorption from tricalcium phosphate is considerably less than milk, while calcium carbonate is equivalent to milk.

Magnesium is another essential micronutrient contained in milk [4]. Considering the studied beverages in which the magnesium data were provided (ranging from 0.84 to $10,178.60 \mathrm{mg} / 100 \mathrm{~mL})$, most of them $(87.50 \%, n=14)$ have higher magnesium content than cow's milk (13.35 mg/100 mL). The sapucaia nut-based beverages have a very high magnesium content per $100 \mathrm{~mL}$, exceeding the Tolerable Upper Intake Level (UL) for this nutrient [77]. Therefore, as previously mentioned, these beverages can be used as ingredients in other food products for nutritional purposes [47].

Iron is not naturally contained in appreciable amounts in cow's milk [71]. Considering iron content of the beverages that present these data (ranging from 0.04 to $1.40 \mathrm{mg} / 100 \mathrm{~mL}$ ), only a rice-based beverage $(12.50 \%, n=1)$ [46] presented lower amount, however close, than the whole cow's milk $(0.05 \mathrm{mg} / 100 \mathrm{~mL})$. With that, plant-based beverages have a certain advantage in terms of quantity, mainly considering a vegetarian diet that, in general, lacks iron [78].

Sodium is a component of salt and it is also found in milk [28]. Craig and Fresán [20] pointed out that consumers are generally concerned about the content of this mineral in plant-based beverages for health reasons. The high consumption of this nutrient is associated with noncommunicable diseases [28]. Among the plant-based beverages that present sodium data that ranged from 0.00 to $343.43 \mathrm{mg} / 100 \mathrm{~mL}, 53.57 \%(n=15)$ of the beverages have higher sodium values than cow's milk $(50.32 \mathrm{mg} / 100 \mathrm{~mL})$. These beverages with a higher sodium content (except for the beverages based on sapucaia nut, which went through the process of crioconcentration as previously mentioned [47]), are commercial beverages that have salt as an ingredient [52]. Despite this, other beverages contain salt as an ingredient that has a lower sodium content than this cow's milk [52,55].

Unfortunately, the function of salt in these beverages that present sodium information and have salt as an ingredient has not been reported. However, Karimidastjerd and KilicAkyilmaz [60] reported adding salt to the beverage to balance the flavor, and Pinelli et al. [79] used salt to produce a quinoa-based beverage to increase the protein content.

\subsection{Limitations}

Lastly, the main limitation of this study is that not all studies provided the information in a more homogeneous way, which did not allow the realization of more elaborate statistics and the grouping of data. Additionally, as limitation, the lack of some data on the micronutrients studied and more information on the quality/type of nutrients for all types of beverages studied for a complete analysis.

\section{Conclusions}

The demand for plant-based beverages used as substitutes for cow's milk is increasing worldwide. In that sense, there is a wide variety of these beverages and new ones are constantly emerging. Despite some similarities to cow's milk, such as appearance, this review showed that the nutritional aspects of these beverages vary widely (energy value varied from 6 to $183 \mathrm{Kcal} / 100 \mathrm{~mL}$; carbohydrate from 0.00 to $22.29 \mathrm{~g} / 100 \mathrm{~mL}$; protein from 0.06 to $12.43 \mathrm{~g} / 100 \mathrm{~mL}$; lipid from 0.00 to $19.00 \mathrm{~g} / 100 \mathrm{~mL}$; dietary fiber from 0.00 to 
$4.40 \mathrm{~g} / 100 \mathrm{~mL}$; calcium from 0.00 to $1252.94 \mathrm{mg} / 100 \mathrm{~mL}$; iron from 0.04 to $1.40 \mathrm{mg} / 100 \mathrm{~mL}$; magnesium from 0.84 to $10,178.60 \mathrm{mg} / 100 \mathrm{~mL}$; sodium from 0.00 to $343.43 \mathrm{mg} / 100 \mathrm{~mL}$ ).

Our initial hypothesis, that plant-based beverages are lower in protein and calcium than cow's milk, was partially confirmed given that some samples presented similar or higher protein and calcium content than cow's milk. A commercial soy-beverage (with apple concentrate, algae Lithothamnium calcareum, and salt) and sapucaia nut-based beverage (under crioconcentration process) exceeded the amount of protein and calcium compared to cow's milk, proving to be good alternatives regarding these parameters.

Different types of raw material used, the added ingredients, the extraction process, and the treatments were used to improve the nutritional content of plant-based beverages aiming to be healthier and similar to cow's milk. Soy is the most common matrix used in the plant-based beverages studied. Based on the ingredient list of the studied products, salt was the added ingredient that most frequently appeared, followed by sugar. However, considering all the sources of ingredients used to sweeten plant-based beverages, all of them presented a type (or combination) of sweetener ingredient, probably in higher amounts than salt aiming to improve sensory characteristics and acceptance. However, it was not possible to analyze it in this study due to the lack of information in some studies about the quantity of the ingredients. Further studies are necessary to evaluate the amount of each ingredient in plant-based beverages and its impacts on individuals' health.

It is necessary to pay attention to the substitution of cow's milk by these alternatives considering the nutritional quality. Due to the diversity of nutrients' type and amount of nutrients found in the studies analyzed, it is noteworthy that most plant-based beverages cannot completely fulfill the replacement of cow's milk regarding nutritional quality. Some of them present ingredients (legumes, almonds, nuts, seeds, etc.) that can also be allergenic for some individuals and their use should be evaluated with caution for allergic individuals. Therefore, having nutritional monitoring is important for an adequate replacement of cow's milk in the diet. The choice of plant-based beverage will depend on the objective (nutritional or sensory) that the person is looking for to use this product and their preferences/restrictions. Thereby, this study can be useful in choosing the best alternative to compose their diet.

Author Contributions: Conceptualization, I.F., B.R. and R.P.Z.; methodology, I.F., B.R. and R.P.Z.; formal analysis, I.F.; resources, R.P.Z.; data curation, I.F., B.R. and R.P.Z.; writing-original draft preparation, I.F., B.R. and R.P.Z.; writing-review and editing, B.R., H.H., A.R. and R.P.Z.; visualization, I.F., B.R., A.R. and R.P.Z.; supervision, B.R., R.P.Z., A.R., H.H., A.A.-M. and L.A.-C.; funding acquisition, H.H., A.R., A.A.-M. and L.A.-C. All authors have read and agreed to the published version of the manuscript.

Funding: This research received no external funding.

Institutional Review Board Statement: Not applicable.

Informed Consent Statement: Not applicable.

Data Availability Statement: This study did not report any data.

Conflicts of Interest: The authors declare no conflict of interest.

Appendix A. Search Terms Used to Perform the Literature Search

Milk alternative; Milk substitute; Plant-based milk; Plant-based beverage; Plant-based drink; Plant-based alternative; Milk analog; Non-dairy milk; Non-dairy beverages; Non-dairy alternative; Nondairy beverage; Plant milk; Vegan milk; Vegetable milk; Plant-based dairy

Portuguese Extrato vegetal; Extrato hidrossolúvel; Bebida à base de 


\section{Appendix B. Table of Authors, Year of Publication and Country of Origin of the Selected Studies According to the Inclusion and Exclusion Criteria}

\begin{tabular}{|c|c|c|}
\hline Authors & Year of Publication & Country of Origin \\
\hline Abrão [30] & 2019 & Brazil \\
\hline Alozie; Udofia [58] & 2015 & Nigeria \\
\hline Andrade [31] & 2018 & Brazil \\
\hline Araújo [41] & 2015 & Brazil \\
\hline Barros [43] & 2012 & Brazil \\
\hline Barros [42] & 2016 & Brazil \\
\hline Barros; Venturini Filho [44] & 2016 & Brazil \\
\hline Blum et al. [45] & 2016 & Brazil \\
\hline Carvalho et al. [46] & 2011 & Brazil \\
\hline Chalupa-Krebzdak et al. [23] & 2018 & Canada \\
\hline Decloedt et al. [52] & 2018 & Belgium \\
\hline Demoliner [47] & 2019 & Brazil \\
\hline Ferreira [48] & 2011 & Brazil \\
\hline Hajirostamloo [56] & 2009 & Iran \\
\hline Holanda [32] & 2017 & Brazil \\
\hline Jeske [57] & 2018 & Ireland \\
\hline $\begin{array}{l}\text { Karimidastjerd; } \\
\text { Kilic-Akyilmaz [60] }\end{array}$ & 2021 & Turkey \\
\hline Lima et al. [33] & 2020 & Brazil \\
\hline Manassero et al. [51] & 2020 & Argentina \\
\hline Manzoor et al. [59] & 2017 & Pakistan \\
\hline Martínez-Padilla et al. [53] & 2020 & Denmark \\
\hline Meeshi et al. [49] & 2014 & India \\
\hline Nti et al. [55] & 2016 & Ghana \\
\hline Ravindran; RadhaiSri [50] & 2020 & India \\
\hline Reis [34] & 2019 & Brazil \\
\hline Scholz-Ahrens et al. [54] & 2020 & Germany \\
\hline Silva et al. [35] & 2015 & Brazil \\
\hline Silva [36] & 2018 & Brazil \\
\hline Storck; Montagner [37] & 2020 & Brazil \\
\hline Uliana; Venturini Filho [38] & 2010 & Brazil \\
\hline Vieira [39] & 2013 & Brazil \\
\hline Vieira [40] & 2017 & Brazil \\
\hline
\end{tabular}

\section{References}

1. $\quad$ FDA. CFR—Code of Federal Regulations Title 21; FDA: Silver Spring, MD, USA, 2018.

2. FAO. Milk and Milk Products; FAO: Rome, Italy, 2011.

3. Siqueira, K.B. O Mercado Consumidor de Leite e Derivados; Embrapa Gado de Leite: Juiz de Fora, Brazil, 2019.

4. Araújo, W.; Montebello, N.; Botelho, R.; Borgo, L. Alquimia dos Alimentos, 3rd ed.; Senac-DF: Brasília, Brazil, 2014.

5. Silva, A.R.A.; Silva, M.M.N.; Ribeiro, B.D. Health Issues and Technological Aspects of Plant-based Alternative Milk. Food Res. Int. 2019, 131, 108972. [CrossRef]

6. Vanga, S.K.; Raghavan, V. How well do plant based alternatives fare nutritionally compared to cow's milk? J. Food Sci. Technol. 2018, 55, 10-20. [CrossRef]

7. Flom, J.D.; Sicherer, S.H. Epidemiology of cow's milk allergy. Nutrients 2019, 11, 1051. [CrossRef]

8. Solé, D.; Silva, L.R.; Cocco, R.R.; Ferreira, C.T.; Sarni, R.O.; Oliveira, L.C.; Pastorino, A.C.; Weffort, V.; Morais, M.B.; Barreto, B.P.; et al. Consenso Brasileiro sobre Alergia Alimentar: 2018-Parte 1-Etiopatogenia, clínica e diagnóstico. Arq. Asma Alerg. Imunol. 2018, 2, 7-38.

9. Friedrich, D.C. A Diversidade do Gene LCT e a Persistência da Lactase na População Brasileira. Ph.D. Thesis, Universidade Federal do Rio Grande do Sul, Porto Alegre, Brazil, 2013.

10. Munekata, P.E.S.; Domínguez, R.; Budaraju, S.; Roselló-Soto, E.; Barba, F.J.; Mallikarjunan, K.; Roohinejad, S.; Lorenzo, J.M. Effect of innovative food processing technologies on the physicochemical and nutritional properties and quality of non-dairy plant-based beverages. Foods 2020, 9, 288. [CrossRef] [PubMed]

11. Mäkinen, O.E.; Wanhalinna, V.; Zannini, E.; Arendt, E.K. Foods for Special Dietary Needs: Non-dairy Plant-based Milk Substitutes and Fermented Dairy-type Products. Crit. Rev. Food Sci. Nutr. 2016, 56, 339-349. [CrossRef] [PubMed]

12. National Institute of Health Lactose intolerance: Genetics Home Reference. Available online: https://ghr.nlm.nih.gov/condition/ lactose-intolerance (accessed on 20 August 2020).

13. Slywitch, E. Guia alimentar de Dietas Vegetarianas; Departamento de Medicina e Nutrição—Sociedade Vegetariana Brasileira, Ed.; Sociedade Brasileira Vegetariana: São Paulo, Brazil, 2012. 
14. Paul, A.A.; Kumar, S.; Kumar, V.; Sharma, R. Milk Analog: Plant based alternatives to conventional milk, production, potential and health concerns. Crit. Rev. Food Sci. Nutr. 2019, 60, 3005-3023. [CrossRef]

15. Haenlein, G.F.W. Goat Milk in Human Nutrition. Small Rumin. Res. 2004, 51, 155-163. [CrossRef]

16. Sarti, L.; Martini, M.; Brajon, G.; Barni, S.; Salari, F.; Altomonte, I.; Ragona, G.; Mori, F.; Pucci, N.; Muscas, G.; et al. Donkey's Milk in the Management of Children with Cow's Milk protein allergy: Nutritional and hygienic aspects. Ital. J. Pediatr. $2019,45,102$. [CrossRef]

17. Li, Y.; Fan, Y.; Shaikh, A.S.; Wang, Z.; Wang, D.; Tan, H. Dezhou Donkey (Equus asinus) Milk a Potential Treatment Strategy for Type 2 Diabetes. J. Ethnopharmacol. 2020, 246, 112221. [CrossRef]

18. He, M.; Sun, J.; Jiang, Z.Q.; Yang, Y.X. Effects of Cow's Milk Beta-Casein Variants on Symptoms of Milk Intolerance in Chinese Adults: A Multicentre, Randomised Controlled Study. Nutr. J. 2017, 16, 72. [CrossRef] [PubMed]

19. Sethi, S.; Tyagi, S.K.; Anurag, R.K. Plant-based milk alternatives an emerging segment of functional beverages: A review. J. Food Sci. Technol. 2016, 53, 3408-3423. [CrossRef]

20. Craig, W.J.; Fresán, U. International analysis of the nutritional content and a review of health benefits of non-dairy plant-based beverages. Nutrients 2021, 13, 842. [CrossRef] [PubMed]

21. Damasceno, L.R.A.D.; Botelho, R.B.A.; de Alencar, E.R. Development of novel plant-based milk based on chickpea and coconut. LWT 2020, 128, 109479. [CrossRef]

22. McClements, D.J. Development of Next-Generation Nutritionally Fortified Plant-Based Milk Substitutes: Structural Design Principles. Foods 2020, 9, 421. [CrossRef]

23. Chalupa-krebzdak, S.; Long, C.J.; Bohrer, B.M. Nutrient density and nutritional value of milk and plant-based milk alternatives. Int. Dairy J. 2018, 87, 84-92. [CrossRef]

24. Cordova, A.G. Consumo de Bebidas Vegetais no Brasil: Análise da Percepção do Consumidor, Pelo Uso de Word Association. Bachelor's Thesis, Universidade Federal de Santa Catarina, Florianópolis, Brazil, 2019.

25. Révillion, J.P.; Kapp, C.; Badejo, M.S.; da Veiga Dias, V. O mercado de alimentos vegetarianos e veganos: Características e perspectivas. Cad. Ciênc. Tecnol. 2020, 37, 26603. [CrossRef]

26. Research and Markets. Global Plant Based Milk Market (Soy Milk, Almond Milk and Rice Milk): Insights, Trends and Forecast (2020-2024); Research and Markets: Dublin, Ireland, 2020.

27. Aqua-Calc Online Food Calculator. Food Volume to Weight Conversions-Soymilk. Available online: https://www.aqua-calc. $\mathrm{com} /$ (accessed on 4 March 2021).

28. WHO. Guideline: Sodium Intake for Adults and Children; WHO: Geneva, Switzerland, 2012; pp. 1-56.

29. Zygomatic Wordclouds. Wordclouds Home Page. Available online: https://www.wordclouds.com/ (accessed on 20 April 2021).

30. Abrão, Y.B. Avaliação Físico-Química de Extratos de Arroz Polido, Parboilizado, Integral e Vermelho; Instituto Federal Goiano-Campus Morrinhos: Morrinhos, Brazil, 2019.

31. Andrade, E.D.d.O. Extrato de Aveia (Avena sativa L.): Obtenção, Determinação da Composição Centesimal e Avaliação Sensorial. Undergraduate Thesis, Federal University of Rio Grande do Norte, Natal, Brazil, 2018.

32. Holanda, S.A.d.M. Desenvolvimento e Caracterização de Bebida Vegetal à Base de Amêndoa de Castanha de Caju, Adicionada de Achocolatado, Leite de Coco ou Banana. Master's Thesis, Universidade Federal do Ceará, Fortaleza, Brazil, 2017.

33. Lima, J.R.; Bruno, L.M.; Wurlitzer, N.J.; de Sousa, P.H.M.; Samara Alves de Mesquita, H. Cashew nut-based beverage: Development, characteristics and stability during refrigerated storage. Food Sci. Technol. 2020, 41, 60-64. [CrossRef]

34. Reis, C.S. dos Estudo do Processamento e Caracterização Físico-Química da Bebida de Gergelim. Undergraduate Thesis, Federal University of Maranhão, São Luís, Brazil, 2019.

35. Silva, L.H.M.; Rodrigues, A.M.C.; Amante, E.R.; Pinheiro, R.C. Caracterização química da amêndoa de frutos amazônicos e seu aproveitamento na elaboração de extratos. In Anais do XX Congresso Brasileiro de Engenharia Química—COBEQ 2014; Blucher: São Paulo, Brazil, 2015; Volume 1, pp. 1-8.

36. Silva, N.L.d.N. Obtenção e composição centesimal de extrato vegetal de amêndoas como alternativa de uso em preparações para indivíduos com intolerância à lactose. Undergraduate Thesis, Federal University of Rio Grande do Norte, Natal, Brazil, 2018.

37. Storck, C.R.; Montagner, G.E. Sorvete com extrato hidrossolúvel de arroz: Análise físico-química e sensorial. DEMETRA Aliment. Nutr. Saúde 2020, 15, e45766. [CrossRef]

38. Uliana, M.R.; Venturini Filho, W.G. Análise Energética De Bebida Mista De Extrato Hidrossolúvel De Soja E Suco De Amora. Energy Agric. 2010, 25, 94-103. [CrossRef]

39. Vieira, A.R. Efeito da Força Iônica na Composição da Bebida à Base de Quinoa Real. Bachelor's Thesis, Universidade de Brasília, Brasília, Brazil, 2013.

40. Vieira, C.F.d.S. Elaboração e Caracterização de Iogurte de Extrato Hidrossolúvel da Amêndoa de Baru (Dipterix Alata vog.). Master's Thesis, Federal University of Tocantins, Palmas, Brazil, 2017.

41. Araújo, A.R. Pastel Sem Glúten e Sem Leite: Uma Alternativa às Restrições Alimentares. Bachelor's Thesis, Universidade de Brasília, Brasília, Brazil, 2015.

42. Barros, É.A. Produção de Bebida Mista de Extrato Hidrossolúvel de Soja e Suco de Uva Submetida a Diferentes Doses de Radiação Gama. Ph.D. Thesis, Universidade Estadual Paulista Júlio de Mesquita Filho, São Paulo, Brazil, 2016.

43. Barros, É.A. Estudo de Lipoxigenases em Extrato Hidrossolúvel de Soja (Glycine max (L.) Merr.) Submetido a Diferentes Tratamentos. Master's Thesis, Universidade Estadual Paulista Júlio de Mesquita Filho, São Paulo, Brazil, 2012. 
44. Barros, É.A.; Venturini Filho, W.G. Caracterização físico-química e sensorial de extrato hidrossolúvel de soja obtido por diferentes métodos de processamento. Rev. Bras. Tecnol. Agroind. 2016, 10, 2038-2051. [CrossRef]

45. Blum, J.E.S.; Ramoni, E.O.; Balbi, M.E. Elaboração de extrato hidrossolúvel (leite) a partir de semente de girassol germinada (Helianthus annus L., Asteraceae) e avaliação de sua composição nutricional. Visão Acad. 2016, 17, 81-95.

46. De Carvalho, W.T.; dos Reis, R.C.; Velasco, P.; Soares Júnior, M.S.; Bassinello, P.Z.; Caliari, M. Características Físico-Químicas De Extratos De Arroz Integral, Quirera De Arroz E Soja. Pesqui. Agropecu. Trop. 2011, 41, 422-429. [CrossRef]

47. Demoliner, F. Perfil Químico da Castanha de Sapucaia (Lecythis Pisonis Cambess) e Obtenção de Extrato Hidrossolúvel Vegetal Por Crioconcentração. Master's Thesis, Universidade Federal de Santa Catarina, Florianópolis, Brazil, 2019.

48. Ferreira, J.C. Processos para o Desenvolvimento da Umbuzada em Pó Liofilizada, Composta de Polpa de Umbu, Extrato de Soja e Rapadura. Ph.D. Thesis, Federal University of Campina Grande, Campina Grande, Brazil, 2011.

49. Meeshi, A.; Hiremath, U.; Kundgol, N.G. Nutritive value of safflower and groundnut milk and their products. Int. J. Farm Sci. 2014, 4, 172-176.

50. Ravindran, S.; RadhaiSri, S. Probiotic oats milk drink with microencapsulated Lactobacillus plantarum-An alternative to dairy products. Nutr. Food Sci. 2020, 51, 471-482. [CrossRef]

51. Manassero, C.A.; Añón, M.C.; Speroni, F. Development of a High Protein Beverage Based on Amaranth. Plant Foods Hum. Nutr. 2020, 75, 599-607. [CrossRef]

52. Decloedt, A.I.; Van Landschoot, A.; Watson, H.; Vanderputten, D.; Vanhaecke, L. Plant-based beverages as good sources of free and glycosidic plant sterols. Nutrients 2018, 10, 21. [CrossRef]

53. Martínez-Padilla, E.; Li, K.; Blok Frandsen, H.; Skejovic Joehnke, M.; Vargas-Bello-Pérez, E.; Lykke Petersen, I. In Vitro Protein Digestibility and Fatty Acid Profile of Commercial Plant-Based Milk Alternatives. Foods 2020, 9, 1784. [CrossRef]

54. Scholz-Ahrens, K.E.; Ahrens, F.; Barth, C.A. Nutritional and health attributes of milk and milk imitations. Eur. J. Nutr. 2020, 59, 19-34. [CrossRef]

55. Nti, C.A.; Plahar, W.A.; Annan, N.T. Development and quality characteristics of shelf-stable soy-agushie: A residual by-product of soymilk production. Food Sci. Nutr. 2016, 4, 315-321. [CrossRef] [PubMed]

56. Hajirostamloo, B. Comparison of Nutritional and Chemical Parameters of Soymilk and Cow milk. World Acad. Sci. Eng. Technol. 2009, 57, 436-438.

57. Jeske, S. Evaluation and Improvement of Technological and Nutritional Properties of Plant-Based Milk Substitutes. Ph.D. Thesis, University College Cork, Cork, Ireland, 2018.

58. Alozie, Y.E.; Udofia, U.S. Nutritional and Sensory Properties of Almond (Prunus amygdalu Var. Dulcis) Seed Milk. World J. Dairy Food Sci. 2015, 10, 117-121. [CrossRef]

59. Manzoor, M.F.; Manzoor, A.; Siddique, R.; Ahmad, N. Nutritional and Sensory Properties of Cashew Seed (Anacardium occidentale) Milk. Mod. Concepts Dev. Agron. 2017, 1,1-4. [CrossRef]

60. Karimidastjerd, A.; Kilic-Akyilmaz, M. Formulation of a low-protein rice drink fortified with caseinomacropeptide concentrate. Food Bioprod. Process. 2021, 125, 161-169. [CrossRef]

61. IBGE. Pesquisa de Orçamentos Familiares 2008-2009: Tabela de Composição Nutricional dos Alimentos Consumidos no Brasil; IBGE: Rio De Janeiro, Brazil, 2011; pp. 1-351.

62. Núcleo de Estudos e Pesquisas em Alimentação (NEPA); Universidade Estadual de Campinas (UNICAMP). Tabela Brasileira de Composição de Alimentos. Available online: https:/ /www.cfn.org.br/wp-content/uploads/2017/03/taco_4_edicao_ampliada_ e_revisada.pdf (accessed on 15 May 2020).

63. Munu, N.; Kigozi, J.; Zziwa, A.; Kambugu, R.; Wasswa, J.; Tumutegyereize, P. Effect of Ambient-Soaking Time on Soybean Characteristics for Traditional Soymilk Extraction. J. Adv. Food Sci. Technol. 2016, 3, 119-128.

64. Aydar, E.F.; Tutuncu, S.; Ozcelik, B. Plant-based milk substitutes: Bioactive compounds, conventional and novel processes, bioavailability studies, and health effects. J. Funct. Foods 2020, 70, 103975. [CrossRef]

65. Sousa, A.; Kopf-Bolanz, K.A. Nutritional Implications of an Increasing Consumption of Non-Dairy Plant-Based Beverages Instead of Cow's Milk in Switzerland. Adv. Dairy Res. 2017, 5, 1-7. [CrossRef]

66. Jeske, S.; Zannini, E.; Arendt, E.K. Evaluation of Physicochemical and Glycaemic Properties of Commercial Plant-Based Milk Substitutes. Plant Foods Hum. Nutr. 2017, 72, 26-33. [CrossRef]

67. Saeed, M.; Yasmin, I.; Pasha, I.; Randhawa, M.A.; Khan, M.; Shabbir, M.A.; Khan, W.A. Potential application of inulin in food industry; a review. Pak. J. Food Sci. 2015, 25, 110-116.

68. The Cornucopia Institute. "Pouring" Over Plant-Based Beverages. A Consumer's Guide to Identifying the Best Non-Milk Alternatives. Available online: https://www.cornucopia.org/wp-content/uploads/2019/06/PlantBasedBeverageReport.pdf (accessed on 1 April 2021).

69. FAO; WHO. Guidelines on Food Fortification with Micronutrients; WHO: Geneva, Switzerland, 2006; pp. 1-343.

70. Food and Agriculture Organization of the United Nations (FAO); World Health Organization (WHO). Class Names and The International Numbering System for Food Additives. Available online: http:/ / www.fao.org/fao-who-codexalimentarius/shproxy / en /?lnk=1\&url=https $\% 253 \mathrm{~A} \% 252 \mathrm{~F} \% 252$ Fworkspace.fao.org $\% 252 \mathrm{Fsites} \% 252 \mathrm{Fcodex} \% 252 \mathrm{FStandards} \% 252 \mathrm{FCXG} \% 2 \mathrm{~B} 36-$ 1989\%252FCXG_036e.pdf (accessed on 21 April 2021).

71. FAO. Milk and Dairy Products in Human Nutrition; FAO: Rome, Italy, 2013; pp. 1-377. 
72. U.S. Department of Agriculture (USDA). FoodData Central. Available online: https://fdc.nal.usda.gov/ (accessed on 23 March 2021).

73. FAO. Dietary Protein Quality Evaluation in Human Nutrition: Report of an FAO Expert Consultation; FAO: Rome, Italy, 2013 ; Volume 92.

74. Tirapegui, J. Nutrição, Fundamentos e Aspectos Atuais, 3rd ed.; Atheneu: São Paulo, Brazil, 2013.

75. Lordan, R.; Tsoupras, A.; Mitra, B.; Zabetakis, I. Dairy fats and cardiovascular disease: Do we really need to be concerned? Foods 2018, 7, 29. [CrossRef]

76. American Association of Cereal Chemists (AACC). The Definition of Dietary Fiber; AACC: St. Paul, MN, USA, 2001 ; Volume 46.

77. Institute of Medicine (US) Standing Committee on the Scientific Evaluation of Dietary Reference Intakes. Dietary Reference Intakes for Calcium, Phosphorus, Magnesium, Vitamin D, and Fluoride; Institute of Medicine (US) Standing Committee on the Scientific Evaluation of Dietary Reference Intakes: Washington, DC, USA, 1997.

78. Haider, L.M.; Schwingshackl, L.; Hoffmann, G.; Ekmekcioglu, C. The effect of vegetarian diets on iron status in adults: A systematic review and meta-analysis. Crit. Rev. Food Sci. Nutr. 2016, 58, 1359-1374. [CrossRef] [PubMed]

79. Pineli, L.L.O.; Botelho, R.B.A.; Zandonadi, R.P.; Solorzano, J.L.; de Oliveira, G.T.; Reis, C.E.G.; Teixeira, D.D.S. Low glycemic index and increased protein content in a novel quinoa milk. LWT Food Sci. Technol. 2015, 63, 1261-1267. [CrossRef] 